\title{
Nonlinear state-dependent feedback control of a pest-natural enemy system
}

\author{
Yuan Tian - Sanyi Tang - Robert A. \\ Cheke
}

\begin{abstract}
The numbers of pests and of natural enemies released to control them as part of integrated pest management (IPM) strategies are density dependent. Therefore the numbers of natural enemies to be released and the rate at which they kill pests should depend on their densities when the number of the pest population has reached the economic threshold. Bearing this in mind, a classic Lotka-Volterra system but with nonlinear state-dependent feedback control tactics is proposed and analyzed in this paper. Furthermore, the definition and properties of the Poincaré map which is defined in the phase set were investigated for various cases, and all those allow us to address the existence and global stability of an order- 1 periodic solution of the model with nonlinear feedback control. Moreover, the existence and nonexistence of periodic solutions with an order larger than 2 or 3 are also discussed. The modelling methods and analytical techniques developed could be widely used and applied in other systems with threshold control such as the glucose insulin regulatory system.
\end{abstract}

Keywords Nonlinear control · Poincaré map · Order-1 periodic solution . Global stability $\cdot$ Pest control

Yuan Tian · Sanyi Tang ( $ه$ )

School of Mathematics and Information Science, Shaanxi Normal University, Xi'an, 710062, P.R.China

Tel.: +86-29-85310232

Fax: +86-29-85310232

E-mail: sytang@snnu.edu.cn \& sanyitang219@hotmail.com

Yuan Tian

School of Science, Hubei University For Nationalities, Enshi, 445000, P.R.China

E-mail: ty0830@snnu.edu.cn

Robert A. Cheke

Natural Resources Institute, University of Greenwich at Medway, Central Avenue, Chatham Maritime, Chatham, Kent, ME4 4TB, UK

E-mail: R.A.Cheke@greenwich.ac.uk 


\section{Introduction}

The well known Lotka-Volterra system, which describes an ecological relationship between two species, plays a key role in theoretical ecology. Volterra first explained oscillations in the volumes of fish catches in the Finme harbour of Italy during the First World War with his model, which later led to what is now known as the Volterra principle[1]. Many scholars have studied the model, by taking into account more realistic factors than he did, and the structure of the Lotka-Volterra system has been much improved and extended[2-7]. More species can be introduced into the model, for which methods used in the two-dimensional system are extended to an n-dimensional system [8, 9]. A time-delay effect has also been considered[10-14], as well as the influence of diffusion in the system[15-18].

In such extended models, the Lotka-Volterra predator-prey system with impulsive effects is often used in models of integrated pest management(IPM). IPM is defined as the careful consideration of all available control techniques to eliminate or mitigate economic and plant health damage caused by pests and to minimize risks to human health and the environment[19, 20]. Tang and Chen[21] developed the Lotka-Volterra system by introducing two different types of impulsive effects: fixed moments and unfix moments (so called as state dependent feedback control). In the first model, these authors provided the threshold condition under which the pest-eradication periodic solution is globally stable. A tenet of IPM is to keep the quantity of a pest below a threshold level to prevent ecological damage by, for instance, not overusing pesticides while keeping the pest density below its economic threshold (ET). The second model was constructed according to such requirements, and a stable order-1 periodic solution which is less than the given ET was obtained. The model showed mathematically that the IPM measure was more effective than any single control tactic.

$\operatorname{In}[22]$, the authors developed the analytical techniques to investigate the state-dependent impulsive models of IPM strategies and showed that the proposed simple model can only exist order- 1 or order- 2 periodic solutions. Furthermore, the relations between the existence of an order-2 limit cycle and the existence of an order-1 limit cycle have been discussed. Importantly, the detailed definitions and domains of several positive invariant sets and attractors of the proposed model have been investigated. Moreover, by constructing the suitable Lyapunov functions the authors provided some sufficient conditions under which the order-1 limit cycle is globally stabile.

In the classic Lotka-Volterra model, the relative growth rate of the two species is a linear function, which does not fully describe the interaction of two species. In order to make the model more realistic, the Lotka-Volterra model has been improved and extended by previous scholars [23-30]. In references such as [26] and [27], the authors proposed, respectively, a linear and a nonlinear Holling type II pest and natural enemy system with impulsive effects; A non-autonomous predator-prey Lotka-Volterra system with mixed functional responses and impulsive effects was presented and studied in [28]. 
The key assumptions in all previous models are: (1) the killing efficacy is proportional to the density or number of the pest population no matter how large the economic threshold is; (2) the number of natural enemies to be released at each impulsive event is a constant once the density of the pest population reaches the economic threshold, which indicates that the number of natural enemies to be released is independent of their density. However, IPM programmes require accurate identifications and monitoring of the pest populations, so that appropriate integrated control decisions can be made in conjunction with the economic threshold. For example, the instantaneous killing rate should be varied according to a saturation function which depends strictly on the pest density, while the number of natural enemies to be released should be a function of their density such that the fewer the natural enemies the more that should be released.

Therefore, in order to address how the density dependent integrated control or nonlinear control actions influence the dynamic behavior of a system with state dependent feedback control, and consequently affect the biological outcomes, we propose the following novel mathematical model:

$$
\left\{\begin{aligned}
\frac{d x(t)}{d t} & =x(t)\left[a-\frac{x(t)}{K}-b y(t)\right], \\
\frac{d y(t)}{d t} & =y(t)(c x(t)-d), \\
x\left(t^{+}\right) & =\left[1-\frac{\delta x(t)}{x(t)+\beta}\right] x(t), \\
y\left(t^{+}\right) & =y(t)+\frac{\tau}{1+\theta y(t)},
\end{aligned}\right\} x(t)=V_{L} .
$$

Model (1) without integrated control measures is a classic Lotka-Volterra system, which has been widely used to depict the interaction between the pest $(x(t))$ and natural enemy $(y(t))$ populations, and the biological meanings of each term can be found in[1,22]. The discrete maps shown in the third and fourth equations represent how to implement the integrated control interventions which depends on the threshold level $V_{L}$, i.e. the controlling strategies should be applied immediately and the numbers of the pest and its natural enemy are updated to $\left(1-\frac{\delta x(t)}{x(t)+\beta}\right) x(t)$ and $y(t)+\frac{\tau}{1+\theta y(t)}\left(\right.$ i.e. $x\left(t^{+}\right)$and $\left.y\left(t^{+}\right)\right)$, respectively.

We assume that $x\left(0^{+}\right)$and $y\left(0^{+}\right)$are the initial densities of the pest and natural enemy populations after the control action is applied at time zero, which satisfy $x\left(0^{+}\right)<V_{L}$ and $y\left(0^{+}\right)>0$. Here the parameters $\delta>0$ and $\beta>0$ denote the maximal killing rate once the pesticide is applied and the half saturation constant, respectively, $\tau>0$ represents the maximum number of predators released, and $\theta>0$ is a shape parameter. Moreover, the nonlinear releasing factor $\frac{\tau}{1+\theta y(t)}$ is a decreasing function of $y(t)$, which indicates that the number of natural enemies released depends on their density and the maximum number of natural enemies to be released is not more than $\tau$ according to some realistic factors including limited resources. Note that by using notations and 
definitions similar to those in reference[26] we can define model (1) as an impulsive semi-dynamical system.

The main purpose of this paper is to investigate the global dynamical behaviour of system (1), reveal how nonlinear impulsive control actions influence the global dynamics and address the biological implications. Firstly, the main properties of an ODE model will be introduced in Section 2. We characterize the impulsive and phase sets and discuss the definition of the Poincaré map by employing the properties of phase portraits of the proposed model and the Lambert $\mathrm{W}$ function in Section 3. In Section 4, sufficient conditions for the existence and global stability of the order-1 limit cycles for $\tau=0$ are given. Moreover, in Section 5, we first investigate the existence and stability of order1 limit cycles for some special cases, and then we further discuss whether an order- $k(k \geq 2)$ periodic solution exists or not. Finally, our conclusions and biological discussion are provided in Section 6 .

\section{The ODE model}

In order to compare results from our new model with the main results obtained in Tang and Cheke [22], we assume that the carrying capacity $K$ is infinite, i.e. the pest population can grow exponentially in the absence of the natural enemy. This is realistic for the initial stage of the pest population growth, i.e. we could assume that the pest population grows very fast before implementing IPM. Thus, the ODE model considered here is the classic Lotka-Volterra predator-prey model

$$
\left\{\begin{array}{l}
\frac{d x(t)}{d t}=x(t)(a-b y(t)), \\
\frac{d y(t)}{d t}=y(t)(c x(t)-d) .
\end{array}\right.
$$

Scholars have worked a lot on this system, and some important conclusions have been obtained. Thus, we only list the main results as follows[22]:

(1) there exist two equilibria: $O(0,0)$ is a saddle point, and $E_{0}\left(x^{*}, y^{*}\right)=\left(\frac{d}{c}, \frac{a}{b}\right)$ is a stable centre;

(2) the closed trajectories in the first quadrant are contained inside the point $\left(x^{*}, y^{*}\right)$;

(3) the model has the first integral:

$$
H(x, y)=a \ln y-b y-c x+d \ln x=h,
$$

here $h$ is a constant.

The equation $H(x, y)=h$ can be analytically solved with respect to variable $y$ by employing Lambert $\mathrm{W}$ function[22, 31], i.e. we have following two real roots:

$$
y_{L}=-\frac{a}{b} W\left[-\frac{b}{a} \exp \left(-\frac{d}{a} \ln x+\frac{h}{a}+\frac{c x}{a}\right)\right]
$$


and

$$
y_{U}=-\frac{a}{b} W\left[-1,-\frac{b}{a} \exp \left(-\frac{d}{a} \ln x+\frac{h}{a}+\frac{c x}{a}\right)\right] .
$$

Besides, based on the properties of the Lambert $W$ function we can see that both $y_{L}$ and $y_{U}$ are well defined if and only if the following inequality holds

$$
-\frac{b}{a} \exp \left(-\frac{d}{a} \ln x+\frac{h}{a}+\frac{c x}{a}\right) \geq-e^{-1} .
$$

Firstly, we focus on the following equation:

$$
-\frac{d}{a} \ln x+\frac{h}{a}+\frac{c x}{a}=\ln \left[\frac{a}{b} e^{-1}\right],
$$

i.e.

$$
c x+h=d \ln x+a \ln \left[\frac{a}{b} e^{-1}\right]
$$

Denote

$$
\begin{gathered}
F(x)=d \ln x+a \ln \left[\frac{a}{b} e^{-1}\right]-c x-h, \\
F_{1}(x)=d \ln x+a \ln \left[\frac{a}{b} e^{-1}\right], \quad F_{2}(x)=c x+h .
\end{gathered}
$$

Thus, if $F(x) \geq 0$ (i.e. $F_{1}(x) \geq F_{2}(x)$ ), then both $y_{L}$ and $y_{U}$ can be well defined. Furthermore, we have $\lim _{x \rightarrow 0^{+}} F_{1}(x)=-\infty, F_{1}^{\prime}(x)=\frac{d}{x}>0(x>0)$, and the function $F_{1}(x)$ is a monotonically increasing function for $x>0$. Further, solving $F_{1}^{\prime}(x)=F_{2}^{\prime}(x)$ with respect to $x$ yields root $x=x^{*}=\frac{d}{c}$, which happens to be the abscissa of the interior equilibrium $E_{0}$. Therefore, the two functions $F_{1}(x)$ and $F_{2}(x)$ are tangent at point $x=x^{*}$, i.e. $h=h^{*}$ with

$$
h^{*}=a\left(\ln \left(\frac{a}{b}\right)-1\right)+d\left(\ln \left(\frac{d}{c}\right)-1\right) .
$$

Denote

$$
\Gamma_{h}=\{(x, y) \mid H(x, y)=h\},
$$

and $\Gamma_{h}$ converges to the equilibrium $E_{0}$ as $h \rightarrow h^{*}$.

If $h<h^{*}$, then the two functions $F_{1}(x)$ and $F_{2}(x)$ can intersect at two points, with the horizontal coordinates of the two points denoted by $x_{\min }$, $x_{\max }$, as shown in Fig.1. It is easy to see that the two curves $y_{L}$ and $y_{U}$ can be well defined for all $x \in\left[x_{\min }, x_{\max }\right]$ with $y_{L} \leq \frac{a}{b} \leq y_{U}$.

Meanwhile, the solution $(x(t), y(t))$ starting from $\left(x_{0}, y_{0}\right)$ satisfies the following relation

$$
\int_{x_{0}}^{x}\left(c-\frac{d}{z}\right) d z=\int_{y_{0}}^{y}\left(\frac{a}{z}-b\right) d z,
$$

i.e.

$$
a \ln y-b y+d \ln x-c x=h_{0}
$$

with $h_{0}=a \ln y_{0}-b y_{0}-c x_{0}+d \ln x_{0}$. 


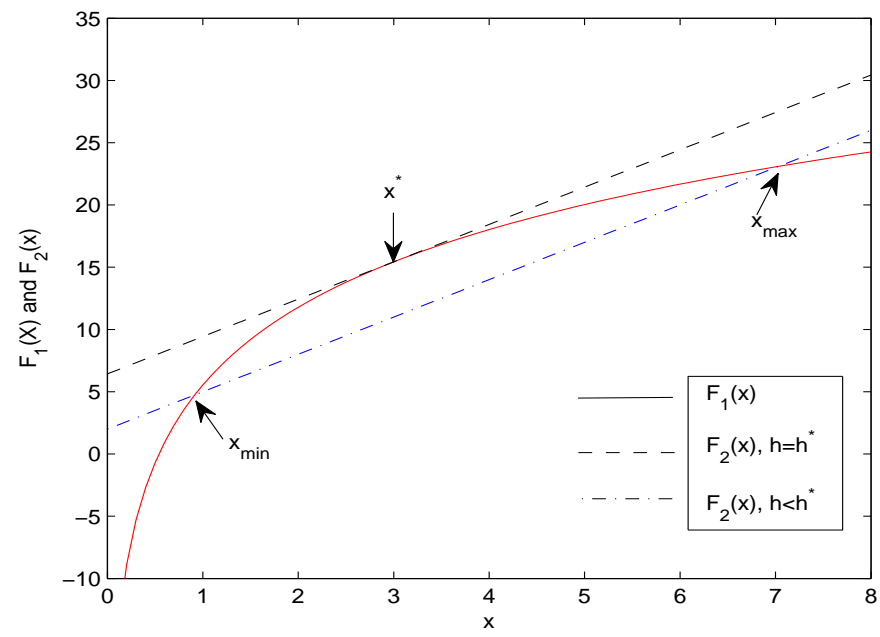

Fig. 1 The existence of the positive roots of $F_{1}(x)=F_{2}(x)$ as $h$ varies, where parameter values are fixed as follows: $a=4, b=e^{-1}, c=3, d=9, h^{*}=\ln \left(4^{4} \cdot 3^{9}\right)-9$.

\section{Analytical formula for the Poincaré map}

In order to obtain the analytical formula for the Poincaré map and investigate the dynamics of model (1), we first discuss the exact domain of the impulsive and phase sets, which play a key role in defining the Poincaré map.

The solution of system (1) initiating from $\left(x_{0}^{+}, y_{0}^{+}\right) \in \mathcal{N}$ may be free from pulse perturbations, or experiences finitely many impulsive effects or infinitely many impulsive effects [26]. For example, any solution starting from the interior of segment $\overline{P_{1} P_{2}}$ does not experience any impulsive effects and any solution initiating from $L_{3} / \overline{P_{1} P_{2}}$ will experience at least one impulsive effect, which depends on the impulsive functions, as shown in Fig.2(A). However, if the threshold value $V_{L}$ satisfies the conditions shown in Fig.2(B), then any solution initiating from $L_{3}$ will experience infinitely many impulsive effects. Therefore, what we want to know first is that the trajectory of system (1) initiating from $\left(x_{0}^{+}, y_{0}^{+}\right) \in \mathcal{N}$ cannot reach the segments of the maximal impulsive set $\mathcal{M}=\left\{(x, y) \mid x=V_{L}, 0 \leq y \leq \frac{a}{b}\right\}$. To address this, based on the relations between $V_{L}$ and $x^{*}$ we consider two possible cases as follows:

$$
\left(A_{1}\right) V_{L}>x^{*} ; \quad\left(A_{2}\right) V_{L} \leq x^{*} .
$$

Moreover, there exists a critical value determined by the parameters of model (1), denoted by $A_{h}$, i.e. we have

$$
A_{h}=d \ln \left(1-\frac{\delta V_{L}}{V_{L}+\beta}\right)+c \delta \frac{V_{L}^{2}}{V_{L}+\beta},
$$

which is important for depicting the dynamics of the model. Denote the point $E_{1}=\left(x_{1}, \frac{a}{b}\right)$ as the intersection point of the closed trajectory $\Gamma_{h}$ with the line 
$y=\frac{a}{b}$ (denoted by $L_{1}$ ) and $E_{0}\left(x^{*}, y^{*}\right) \in \operatorname{Int} \Gamma_{h}$, and $\Gamma_{h}$ is tangent to the line $x=V_{L}$ (denoted by $\left.L_{2}\right)$ at point $T\left(V_{L}, \frac{a}{b}\right)$, as shown in Fig.2(A). It is easy to see that $x_{1}<x^{*}<V_{L}$. Then, based on the positions of the two lines $x=V_{L}$ and $x=\left(1-\frac{\delta V_{L}}{V_{L}+\beta}\right) V_{L}$ (denoted by $L_{3}$ ), we can address cases of the impulsive and phase sets under different circumstances.

(A)

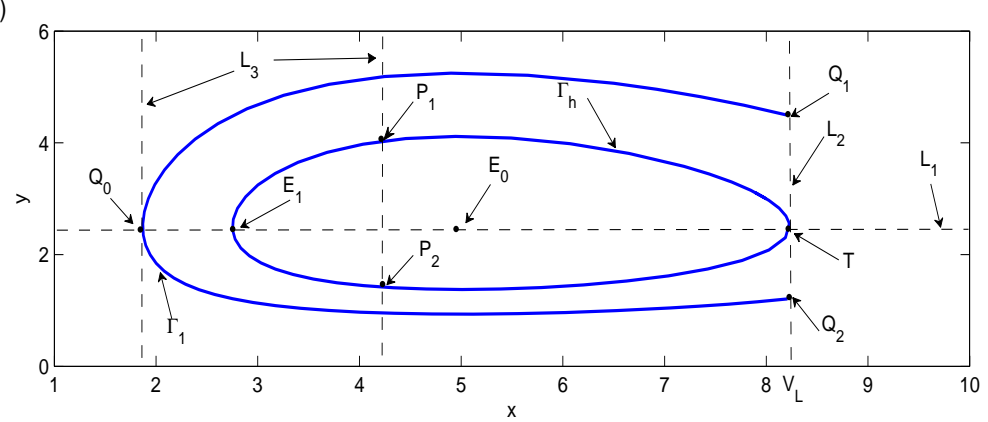

(B)

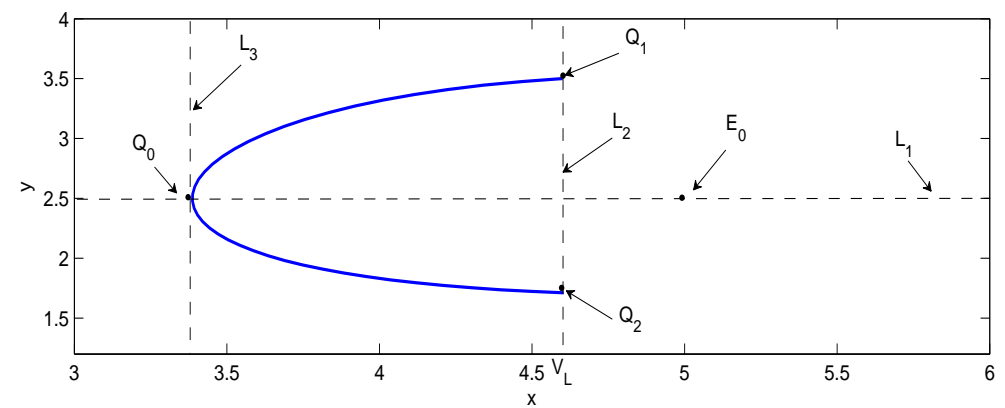

Fig. 2 The position relationship of two lines $L_{2}$ and $L_{3}$ related to the definition of the impulsive and phase sets for cases $\left(A_{1}\right)$ and $\left(A_{2}\right) .(A): V_{L}>x^{*}$ and $\left(1-\frac{\delta V_{L}}{V_{L}+\beta}\right) V_{L} \geq x_{1}$ or $\left(1-\frac{\delta V_{L}}{V_{L}+\beta}\right) V_{L}<x_{1} ;(B): V_{L} \leq x^{*}$ and $\left(1-\frac{\delta V_{L}}{V_{L}+\beta}\right) V_{L}<x^{*}$.

\subsection{Impulsive set}

According to the properties of planar impulsive semi-dynamic systems [32-36], we can discuss the impulsive sets of system (1) as follows.

The basic impulsive set $\mathcal{M}$ defined as

$$
\mathcal{M}=\left\{(x, y) \in R_{+}^{2} \mid x=V_{L}, 0 \leq y \leq \frac{a}{b}\right\}
$$


The set $\mathcal{M}_{0}$ defined as

$$
\mathcal{M}_{0}=\left\{(x, y) \in R_{+}^{2} \mid x=V_{L}, 0 \leq y \leq Y_{i s}^{h}\right\}
$$

$\mathcal{M}_{0}$ is a subset or a segment of the basic impulsive set $\mathcal{M}$, where

$$
Y_{i s}^{h}=-\frac{a}{b} W\left(-e^{-1+\frac{A_{h}}{a}}\right)
$$

with $A_{h} \leq 0$, and note that $\mathcal{M}_{0}=\mathcal{M}$ if $A_{h}=0$.

Lemma 1 For case $\left(A_{1}\right)$, if $\left(1-\frac{\delta V_{L}}{V_{L}+\beta}\right) V_{L} \geq x_{1}$, the impulsive set is $\mathcal{M}$; if $\left(1-\frac{\delta V_{L}}{V_{L}+\beta}\right) V_{L}<x_{1}$, the impulsive set is $\mathcal{M}_{0}$. The impulsive set for case $\left(A_{2}\right)$ is given by $\mathcal{M}_{0}$.

Proof. For case $\left(A_{1}\right)$, if $\left(1-\frac{\delta V_{L}}{V_{L}+\beta}\right) V_{L} \geq x_{1}$, then the trajectory of model (1) initiating from $L_{3}$ can arrive at the set $\mathcal{M}$. If $\left(1-\frac{\delta V_{L}}{V_{L}+\beta}\right) V_{L}<x_{1}$, then there exists a curve $\Gamma_{1}$ which is tangent to the straight line $L_{3}$ at point $Q_{0}$ : $\left(\left(1-\frac{\delta V_{L}}{V_{L}+\beta}\right) V_{L}, \frac{a}{b}\right)$, where the curve $\Gamma_{1}$ satisfies the following equation:

$a \ln y-b y+d \ln x-c x=a \ln \frac{a}{b}-a-c\left(1-\frac{\delta V_{L}}{V_{L}+\beta}\right) V_{L}+d \ln \left(\left(1-\frac{\delta V_{L}}{V_{L}+\beta}\right) V_{L}\right)$.

Note that the intersection points of the straight line $L_{2}$ with the curve $\Gamma_{1}$ can be analytically determined (as shown in Fig. 2(A)), i.e. the vertical components of $Q_{1}$ and $Q_{2}$ can be solved from the following equation:

$$
a \ln y-b y=a \ln \frac{a}{b}-a+A_{h},
$$

i.e.

$$
\left(-\frac{b}{a} y\right) e^{-\frac{b}{a} y}=-e^{-1+\frac{A_{h}}{a}}
$$

If $A_{h}<0$, then we can solve equation (10) with respect to $y$, i.e.

$$
Y_{i s}^{h}=-\frac{a}{b} W\left(-e^{-1+\frac{A_{h}}{a}}\right), \quad Y_{I S}^{h}=-\frac{a}{b} W\left(-1,-e^{-1+\frac{A_{h}}{a}}\right) .
$$

Thus, if $\left(1-\frac{\delta V_{L}}{V_{L}+\beta}\right) V_{L}<x_{1}$, then it is easy to see that there does not exist any solution of model (1) initiating from the line $L_{3}$ that can arrive the set $\left\{(x, y) \mid x=V_{L}, Y_{i s}^{h}<y \leq \frac{a}{b}\right\}$, which indicates that the impulsive set is defined by $\mathcal{M}_{0}$ for case $\left(A_{1}\right)$. By employing the similar methods as those for case $\left(A_{1}\right)$, the impulsive set for case $\left(A_{2}\right)$ can be defined by $\mathcal{M}_{0}$ (as shown in Fig. 2(B)). This completes the proof.

Note that the more detailed definition of the impulsive set should combine with the definition of the phase set, and we will focus on this in the following subsection. 


\subsection{Phase set}

As mentioned before, we must first investigate whether the trajectory of model (1) from the line $L_{3}$ is free from pulse effects or not, which are crucial for defining the impulsive and phase sets, i.e. we have the following main result:

Lemma 2 For case $\left(A_{1}\right)$, if $x_{1} \leq\left(1-\frac{\delta V_{L}}{V_{L}+\beta}\right) V_{L}$, then the solution starting from $\left(x_{0}^{+}, y_{0}^{+}\right) \in L_{3}$ with $y_{0}^{+} \in\left(Y_{\min }^{h}, Y_{\max }^{h}\right)$ does not experience pulse effects, where

$$
Y_{\min }^{h}=-\frac{a}{b} W\left(-e^{-1-\frac{A_{h}}{a}}\right), \quad Y_{\max }^{h}=-\frac{a}{b} W\left(-1,-e^{-1-\frac{A_{h}}{a}}\right) .
$$

Moreover, $x_{1}<\left(1-\frac{\delta V_{L}}{V_{L}+\beta}\right) V_{L} \Leftrightarrow A_{h}>0$, and $A_{h}=0$ at $\left(1-\frac{\delta V_{L}}{V_{L}+\beta}\right) V_{L}=x_{1}$.

Proof. The closed orbit $\Gamma_{h}$ which tangents to the line $L_{2}$ can be analytically determined as follows:

$$
\Gamma_{h}: \quad H(x, y)=a \ln y-b y+d \ln x-c x=a \ln \frac{a}{b}-a-c V_{L}+d \ln V_{L} .
$$

Letting $y=\frac{a}{b}$ in equation (13) yields the following equation

$$
F_{0}(x)=d \ln x-c x+c V_{L}-d \ln V_{L}=0,
$$

and $x_{1}$ is the small root of the above equation.

By simple calculation, we have $F_{0}\left(x_{1}\right)=0, F_{0}\left(V_{L}\right)=0, F_{0}^{\prime}(x)=\frac{d}{x}-c$ and $F_{0}^{\prime}\left(x^{*}\right)=0$. Moreover, $F_{0}^{\prime}(x)>0$ for $x \in\left(x_{1}, x^{*}\right)$, and $F_{0}^{\prime}(x)<0$ for $x \in\left(x^{*}, V_{L}\right)$, which indicates that $F_{0}(x) \geq 0$ for all $x \in\left[x_{1}, V_{L}\right]$.

Note that the line $L_{3}$ intersects with the closed orbit $\Gamma_{h}$ at two points, denoted by $P_{1}=\left(\left(1-\frac{\delta V_{L}}{V_{L}+\beta}\right) V_{L}, Y_{\max }^{h}\right)$ and $P_{2}=\left(\left(1-\frac{\delta V_{L}}{V_{L}+\beta}\right) V_{L}, Y_{\min }^{h}\right)$ (as shown in Fig. 2(A)), where $Y_{\max }^{h}$ and $Y_{\min }^{h}$ are the two roots of equation (13) with $x=\left(1-\frac{\delta V_{L}}{V_{L}+\beta}\right) V_{L}$, i.e. substituting $x=\left(1-\frac{\delta V_{L}}{V_{L}+\beta}\right) V_{L}$ into equation (13) yields

$$
a \ln y-b y=a \ln \frac{a}{b}-a-A_{h}
$$

and further we have

$$
-\frac{b}{a} y e^{-\frac{b}{a} y}=-e^{-1-\frac{A_{h}}{a}} .
$$

If $A_{h} \geq 0$, according to the definition of the Lambert $\mathrm{W}$ function, we can solve (16) with respect to $y$. It follows from $A_{h}=F_{0}\left(\left(1-\frac{\delta V_{L}}{V_{L}+\beta}\right) V_{L}\right) \geq 0$ for all $\left.\left(1-\frac{\delta V_{L}}{V_{L}+\beta}\right) V_{L}\right) \in\left[x_{1}, V_{L}\right]$ that both $P_{1}$ and $P_{2}$ are well defined.

Solving (16) with respect to $y$, yields two roots as follows:

$$
Y_{\min }^{h}=-\frac{a}{b} W\left(-e^{-1-\frac{A_{h}}{a}}\right), \quad Y_{\max }^{h}=-\frac{a}{b} W\left(-1,-e^{-1-\frac{A_{h}}{a}}\right) .
$$

Therefore, the solution starting from $\left(x_{0}^{+}, y_{0}^{+}\right) \in \mathcal{N}$ with $y_{0}^{+} \in\left(Y_{\min }^{h}, Y_{\max }^{h}\right)$ is free from pulse effects. 
Thus, according to the proof of Lemma 2 we can define the following set for case $\left(A_{1}\right)$ and $x_{1} \leq\left(1-\frac{\delta V_{L}}{V_{L}+\beta}\right) V_{L}$ :

$$
Y_{D}^{h}=\left[0, Y_{\min }^{h}\right] \cup\left[Y_{\max }^{h},+\infty\right) .
$$

Moreover, the properties of impulsive function $y\left(t^{+}\right)=y(t)+\frac{\tau}{1+\theta y(t)}$ is also necessary. To do this, denote

$$
G(z)=z+\frac{\tau}{1+\theta z}, \quad z \in\left[0, \frac{a}{b}\right],
$$

and it is easy to see that $G^{\prime}(z)=1-\frac{\tau \theta}{(1+\theta z)^{2}}$ and $G^{\prime}(z)=0$ at $z=\frac{\sqrt{\tau \theta}-1}{\theta}$.

Therefore, according to the sign of $z=\frac{\sqrt{\tau \theta}-1}{\theta}$ and the monotonicity of function $G(z)$, we can discuss the following cases related to the exact impulsive and phase sets.

Case $\left(A_{1}\right): V_{L}>x^{*}$

$\left(A_{11}\right) \quad x_{1} \leq\left(1-\frac{\delta V_{L}}{V_{L}+\beta}\right) V_{L}$, the impulsive set is given as $\mathcal{M}=\{(x, y) \in$ $\left.R_{+}^{2} \mid x=V_{L}, 0 \leq y \leq \frac{a}{b}\right\}$ according to Lemma 1. For the phase set, we consider the following three subcases based on the relations between $\frac{\sqrt{\tau \theta}-1}{\theta}$ and $\frac{a}{b}$.

(i) $\frac{\sqrt{\tau \theta}-1}{\theta} \leq 0$. If so we can show that $G^{\prime}(z) \geq 0$ for all $z \in\left[0, \frac{a}{b}\right]$, which indicates that $\tau \leq G(z) \leq \frac{a}{b}+\frac{\tau}{1+\frac{a}{b} \theta}$. Thus, we denote

$$
Y_{1}^{0}=\left[\tau, \frac{a}{b}+\frac{\tau}{1+\frac{a}{b} \theta}\right], \quad Y_{1}=Y_{D}^{h} \cap Y_{1}^{0} .
$$

Then, the phase set which corresponds to the impulsive set $\mathcal{M}$ is given as

$$
\mathcal{N}_{1}=\left\{\left(x^{+}, y^{+}\right) \in R_{+}^{2} \mid x^{+}=\left(1-\frac{\delta V_{L}}{V_{L}+\beta}\right) V_{L}, y^{+} \in Y_{1}\right\} .
$$

(ii) $\frac{\sqrt{\tau \theta}-1}{\theta} \geq \frac{a}{b}$. Based on this inequality we can show that $G^{\prime}(z) \leq 0$ for $z \in\left[0, \frac{a}{b}\right]$ and $\frac{a}{b}+\frac{\tau}{1+\frac{a}{b} \theta} \leq G(z) \leq \tau$, so according to Lemma 2 we denote

$$
Y_{2}^{0}=\left[\frac{a}{b}+\frac{\tau}{1+\frac{a}{b} \theta}, \tau\right], \quad Y_{2}=Y_{D}^{h} \cap Y_{2}^{0} .
$$

Then, the phase set which corresponds to the impulsive set $\mathcal{M}$ here could be given as

$$
\mathcal{N}_{2}=\left\{\left(x^{+}, y^{+}\right) \in R_{+}^{2} \mid x^{+}=\left(1-\frac{\delta V_{L}}{V_{L}+\beta}\right) V_{L}, y^{+} \in Y_{2}\right\} .
$$

(iii) $0<\frac{\sqrt{\tau \theta}-1}{\theta}<\frac{a}{b}$. If $0 \leq z \leq \frac{\sqrt{\tau \theta}-1}{\theta}$, then we have $G^{\prime}(z) \leq 0$ and $\frac{2 \sqrt{\tau \theta}-1}{\theta} \leq G(z) \leq \tau$, and we denote

$$
Y_{3}^{0}=\left[\frac{2 \sqrt{\tau \theta}-1}{\theta}, \tau\right], \quad Y_{3}=Y_{D}^{h} \cap Y_{3}^{0} .
$$


If $\frac{\sqrt{\tau \theta}-1}{\theta}<z \leq \frac{a}{b}$, then we have $G^{\prime}(z)>0$ and $\frac{2 \sqrt{\tau \theta}-1}{\theta}<G(z) \leq \frac{a}{b}+\frac{\tau}{1+\frac{a}{b} \theta}$, so we denote

$$
Y_{4}^{0}=\left(\frac{2 \sqrt{\tau \theta}-1}{\theta}, \frac{a}{b}+\frac{\tau}{1+\frac{a}{b} \theta}\right], \quad Y_{4}=Y_{D}^{h} \cap Y_{4}^{0} .
$$

Now, the impulsive set $\mathcal{M}$ can be defined as: $\mathcal{M}=\mathcal{M}_{1} \cup \mathcal{M}_{2}$, where

$$
\mathcal{M}_{1}=\left\{(x, y) \in R_{+}^{2} \mid x=V_{L}, 0 \leq y \leq \frac{\sqrt{\tau \theta}-1}{\theta}\right\}
$$

and

$$
\mathcal{M}_{2}=\left\{(x, y) \in R_{+}^{2} \mid x=V_{L}, \frac{\sqrt{\tau \theta}-1}{\theta}<y \leq \frac{a}{b}\right\} .
$$

Therefore, the phase set which corresponds to the impulsive set $\mathcal{M}=\mathcal{M}_{1} \cup$ $\mathcal{M}_{2}$ can be defined as $\mathcal{N}_{3} \cup \mathcal{N}_{4}$, where

$$
\mathcal{N}_{3}=\left\{\left(x^{+}, y^{+}\right) \in R_{+}^{2} \mid x^{+}=\left(1-\frac{\delta V_{L}}{V_{L}+\beta}\right) V_{L}, y^{+} \in Y_{3}\right\}
$$

and

$$
\mathcal{N}_{4}=\left\{\left(x^{+}, y^{+}\right) \in R_{+}^{2} \mid x^{+}=\left(1-\frac{\delta V_{L}}{V_{L}+\beta}\right) V_{L}, y^{+} \in Y_{4}\right\} .
$$

$\left(A_{12}\right) \quad\left(1-\frac{\delta V_{L}}{V_{L}+\beta}\right) V_{L}<x_{1}$. According to Lemma 1 the impulsive set for this subcase can be defined as $\mathcal{M}_{0}=\left\{(x, y) \in R_{+}^{2} \mid x=V_{L}, 0 \leq y \leq Y_{i s}^{h}\right\}$, for the definition of the phase set we further consider the following three subcases.

(i) $\frac{\sqrt{\tau \theta}-1}{\theta} \leq 0$. The phase set which corresponds to $\mathcal{M}_{0}$ here is as follows:

$$
\mathcal{N}_{5}=\left\{\left(x^{+}, y^{+}\right) \in R_{+}^{2} \mid x^{+}=\left(1-\frac{\delta V_{L}}{V_{L}+\beta}\right) V_{L}, \tau \leq y^{+} \leq Y_{i s}^{h}+\frac{\tau}{1+Y_{i s}^{h} \theta}\right\},
$$

and denote $Y_{5}=\left[\tau, Y_{i s}^{h}+\frac{\tau}{1+Y_{i s}^{h} \theta}\right]$.

(ii) $0<\frac{\sqrt{\tau \theta}-1}{\theta}<Y_{i s}^{h}$. For this subcase the impulsive set $\mathcal{M}_{0}$ is given as: $\mathcal{M}_{0}=\mathcal{M}_{1} \cup \mathcal{M}_{3}$, where

$$
\mathcal{M}_{1}=\left\{(x, y) \in R_{+}^{2} \mid x=V_{L}, 0 \leq y \leq \frac{\sqrt{\tau \theta}-1}{\theta}\right\}
$$

and

$$
\mathcal{M}_{3}=\left\{(x, y) \in R_{+}^{2} \mid x=V_{L}, \frac{\sqrt{\tau \theta}-1}{\theta}<y \leq Y_{i s}^{h}\right\} .
$$

Therefore, the phase set which corresponds to the impulsive set $\mathcal{M}_{0}=\mathcal{M}_{1} \cup$ $\mathcal{M}_{3}$ is $\mathcal{N}_{6} \cup \mathcal{N}_{7}$, where

$$
\mathcal{N}_{6}=\left\{\left(x^{+}, y^{+}\right) \in R_{+}^{2} \mid x^{+}=\left(1-\frac{\delta V_{L}}{V_{L}+\beta}\right) V_{L}, \frac{2 \sqrt{\tau \theta}-1}{\theta} \leq y^{+} \leq \tau\right\}
$$


and

$\mathcal{N}_{7}=\left\{\left(x^{+}, y^{+}\right) \in R_{+}^{2} \mid x^{+}=\left(1-\frac{\delta V_{L}}{V_{L}+\beta}\right) V_{L}, \frac{2 \sqrt{\tau \theta}-1}{\theta}<y^{+} \leq Y_{i s}^{h}+\frac{\tau}{1+Y_{i s}^{h} \theta}\right\}$,

and we denote the interval $Y_{6}=\left(\frac{2 \sqrt{\tau \theta}-1}{\theta}, Y_{i s}^{h}+\frac{\tau}{1+Y_{i s}^{h} \theta}\right]$.

(iii) $\frac{\sqrt{\tau \theta}-1}{\theta} \geq Y_{i s}^{h}$. For this subcase the phase set which corresponds to the impulsive set $\mathcal{M}_{0}$ can be defined as

$$
\mathcal{N}_{8}=\left\{\left(x^{+}, y^{+}\right) \in R_{+}^{2} \mid x^{+}=\left(1-\frac{\delta V_{L}}{V_{L}+\beta}\right) V_{L}, Y_{i s}^{h}+\frac{\tau}{1+Y_{i s}^{h} \theta} \leq y^{+} \leq \tau\right\},
$$

and we further denote the interval $Y_{7}=\left[Y_{i s}^{h}+\frac{\tau}{1+Y_{i s}^{h} \theta}, \tau\right]$.

Case $\left(A_{2}\right): V_{L} \leq x^{*}$.

It follows from $V_{L} \leq x^{*}$ and Lemma 1 that for $\left(1-\frac{\delta V_{L}}{V_{L}+\beta}\right) V_{L}<x^{*}$ the impulsive set for case $\left(A_{2}\right)$ is defined as $\mathcal{M}_{0}$. Similarly, the exact phase set can be classified and discussed in the same way as described above, and we have

(i) The phase set is given as $\mathcal{N}_{5}$ for $\frac{\sqrt{\tau \theta}-1}{\theta} \leq 0$.

(ii) The phase set is given as $\mathcal{N}_{6} \cup \mathcal{N}_{7}$ for $0<\frac{\sqrt{\tau \theta}-1}{\theta}<Y_{i s}^{h}$.

(iii) The phase set is given as $\mathcal{N}_{8}$ for $\frac{\sqrt{\tau \theta}-1}{\theta} \geq Y_{i s}^{h}$.

For convenience, we list all possible cases in Table 1, from which we can see clearly how the impulsive set and phase set vary with the key parameters including the threshold value $V_{L}$ and the parameters related to the control actions.

Table 1 Exact domains of the impulsive sets (Is) and phase sets (Ps) of system (1).

\begin{tabular}{|c|c|c|c|c|}
\hline \multicolumn{2}{|c|}{ Cases } & $\left(1-\frac{\delta V_{L}}{V_{L}+\beta}\right) V_{L}$ & Is & Ps \\
\hline \multirow{6}{*}{$\left(A_{1}\right)$} & $(i)$ & \multirow{3}{*}{$\left(1-\frac{\delta V_{L}}{V_{L}+\beta}\right) V_{L} \geq x_{1}$} & \multirow{3}{*}{$\mathcal{M}$} & $\mathcal{N}_{1}$ \\
\hline & (ii) & & & $\mathcal{N}_{2}$ \\
\hline & (iii) & & & $\mathcal{N}_{3} \cup \mathcal{N}_{4}$ \\
\hline & $(i)$ & \multirow{3}{*}{$\left(1-\frac{\delta V_{L}}{V_{L}+\beta}\right) V_{L}<x_{1}$} & \multirow{3}{*}{$\mathcal{M}_{0}$} & $\mathcal{N}_{5}$ \\
\hline & (ii) & & & $\mathcal{N}_{6} \cup \mathcal{N}_{7}$ \\
\hline & (iii) & & & $\mathcal{N}_{8}$ \\
\hline \multirow{3}{*}{$\left(A_{2}\right)$} & (i) & \multirow{3}{*}{$\left(1-\frac{\delta V_{L}}{V_{L}+\beta}\right) V_{L}<x^{*}$} & \multirow{3}{*}{$\mathcal{M}_{0}$} & $\mathcal{N}_{5}$ \\
\hline & (ii) & & & $\mathcal{N}_{6} \cup \mathcal{N}_{7}$ \\
\hline & (iii) & & & $\mathcal{N}_{8}$ \\
\hline
\end{tabular}

\subsection{Poincaré map}

Based on the domains shown in Table 1, we can analytically determine the formula for the Poincaré map, i.e. we have the following main results. 
Theorem 3 In the phase set the Poincaré map of model (1) is analytically given as

Case $\left(A_{1}\right): V_{L}>x^{*}$.

$\left(A_{11}\right)$ For all $x_{1} \leq\left(1-\frac{\delta V_{L}}{V_{L}+\beta}\right) V_{L}$ and $A_{h} \geq 0$ we have

$$
y_{i+1}^{+}= \begin{cases}\mathcal{P}\left(y_{i}^{+}\right), & y_{i}^{+} \in Y_{1}, \quad \text { if } \frac{\sqrt{\tau \theta}-1}{\theta} \leq 0, \\ \mathcal{P}\left(y_{i}^{+}\right), & y_{i}^{+} \in Y_{2}, \quad \text { if } \frac{\sqrt{\tau \theta}-1}{\theta} \geq \frac{a}{b}, \\ \mathcal{P}\left(y_{i}^{+}\right), & y_{i}^{+} \in Y_{3} \cup Y_{4}, \quad \text { if } 0<\frac{\sqrt{\tau \theta}-1}{\theta}<\frac{a}{b} .\end{cases}
$$

$\left(A_{12}\right)$ For all $\left(1-\frac{\delta V_{L}}{V_{L}+\beta}\right) V_{L}<x_{1}$ and $A_{h}<0$ we have

$$
y_{i+1}^{+}= \begin{cases}\mathcal{P}\left(y_{i}^{+}\right), & y_{i}^{+} \in Y_{5}, \text { if } \frac{\sqrt{\tau \theta}-1}{\theta} \leq 0, \\ \mathcal{P}\left(y_{i}^{+}\right), & y_{i}^{+} \in Y_{3}^{0} \cup Y_{6}, \text { if } 0<\frac{\sqrt{\tau \theta}-1}{\theta}<Y_{i s}^{h}, \\ \mathcal{P}\left(y_{i}^{+}\right), & y_{i}^{+} \in Y_{7}, \text { if } \frac{\sqrt{\tau \theta}-1}{\theta} \geq Y_{i s}^{h} .\end{cases}
$$

Case $\left(A_{2}\right): V_{L} \leq x^{*}$. For all $\left(1-\frac{\delta V_{L}}{V_{L}+\beta}\right) V_{L}<x^{*}$ and $A_{h}<0$ we have

$$
y_{i+1}^{+}= \begin{cases}\mathcal{P}\left(y_{i}^{+}\right), & y_{i}^{+} \in Y_{5}, \text { if } \frac{\sqrt{\tau \theta}-1}{\theta} \leq 0, \\ \mathcal{P}\left(y_{i}^{+}\right), & y_{i}^{+} \in Y_{3}^{0} \cup Y_{6}, \text { if } 0<\frac{\sqrt{\tau \theta}-1}{\theta}<Y_{i s}^{h}, \\ \mathcal{P}\left(y_{i}^{+}\right), & y_{i}^{+} \in Y_{7}, \text { if } \frac{\sqrt{\tau \theta}-1}{\theta} \geq Y_{i s}^{h} .\end{cases}
$$

Proof. Without loss of generality, we assume that any solution $\Pi_{z_{0}+}$ initiating from $z_{0}^{+}=\left(x_{0}^{+}, y_{0}^{+}\right) \in \mathcal{N}$ experiences pulse effects $k+1$ times, where constant $k$ could be finite or infinite. Define the point $q_{i}=\left(V_{L}, y_{i}\right) \in \mathcal{M}$ and $q_{i}^{+}=$ $\left(\left(1-\frac{\delta V_{L}}{V_{L}+\beta}\right) V_{L}, y_{i}^{+}\right) \in \mathcal{N}, i=0,1,2, \cdots, k$. The point $q_{i}^{+}$is the resetting point of $q_{i}$. If two points $q_{i}^{+}$and $q_{i+1}$ lie in the trajectory $\Gamma, i=0,1,2, \cdots, k$, then the both points satisfy the following relation:

$$
\int_{V_{L}}^{\left(1-\frac{\delta V_{L}}{V_{L}+\beta}\right) V_{L}}\left(c-\frac{d}{x}\right) d x=\int_{y_{i+1}}^{y_{i}^{+}}\left(\frac{a}{y}-b\right) d y
$$

i.e.

$$
-\frac{b}{a} y_{i+1} \exp \left(-\frac{b}{a} y_{i+1}\right)=-\frac{b}{a} y_{i}^{+} \exp \left(-\frac{b}{a} y_{i}^{+}+\frac{A_{h}}{a}\right),
$$

where $A_{h}=c \delta \frac{V_{L}^{2}}{V_{L}+\beta}+d \ln \left(1-\frac{\delta V_{L}}{V_{L}+\beta}\right)$.

Solving equation (23) with respect to $y_{i+1}$ by using the properties of the Lambert $\mathrm{W}$ function, one has

$$
y_{i+1}=-\frac{a}{b} W\left[-\frac{b}{a} y_{i}^{+} \exp \left(-\frac{b}{a} y_{i}^{+}+\frac{A_{h}}{a}\right)\right] \quad i=0,1,2, \cdots, k
$$

and

$$
y_{i+1}^{+}=y_{i+1}+\frac{\tau}{1+\theta y_{i+1}} \triangleq \mathcal{P}\left(y_{i}^{+}\right) \quad i=0,1,2, \cdots, k
$$


i.e.

$$
\begin{aligned}
y_{i+1}^{+} & =-\frac{a}{b} W\left[-\frac{b}{a} y_{i}^{+} \exp \left(-\frac{b}{a} y_{i}^{+}+\frac{A_{h}}{a}\right)\right]+\frac{\tau}{1-\frac{\theta a}{b} W\left[-\frac{b}{a} y_{i}^{+} \exp \left(-\frac{b}{a} y_{i}^{+}+\frac{A_{h}}{a}\right)\right]} \\
& \triangleq \mathcal{P}\left(y_{i}^{+}\right) \quad i=0,1,2, \cdots, k .
\end{aligned}
$$

Note that the Poincaré map defined in (25) depends on the Lambert $\mathrm{W}$ function and the sign of $A_{h}$. In fact, if $A_{h} \leq 0$, then it is easy to know that $-\frac{b}{a} y_{i}^{+} \exp \left(-\frac{b}{a} y_{i}^{+}+\frac{A_{h}}{a}\right) \in\left[-e^{-1}, 0\right)$. If $A_{h} \geq 0$, then according to results shown in Lemma 2 that for all $y_{i}^{+} \in\left(0, Y_{\min }^{h}\right] \cup\left[Y_{\max }^{h}, \infty\right)$ we have $-\frac{b}{a} y_{i}^{+} \exp \left(-\frac{b}{a} y_{i}^{+}+\frac{A_{h}}{a}\right) \geq-e^{-1}$ due to $-\frac{b}{a} y_{i}^{+} \exp \left(-\frac{b}{a} y_{i}^{+}\right) \geq-\exp \left(-1-\frac{A_{h}}{a}\right)$.

Therefore, the exact domain of the Poincaré map for all cases discussed in Table 1 can be determined accordingly. This completes the proof.

If $\theta=0$ or $\tau=0$, we can obtain the formula of the Poincaré map which has been investigated in [22], i.e. we have the following results.

Corollary 1 If $\theta=0$, the Poincaré map is given as

$$
\mathcal{P}\left(y_{i}^{+}\right)=-\frac{a}{b} W\left[-\frac{b}{a} y_{i}^{+} \exp \left(-\frac{b}{a} y_{i}^{+}+\frac{A_{h}}{a}\right)\right]+\tau .
$$

If $\tau=0$, the Poincaré map is defined as

$$
\mathcal{P}\left(y_{i}^{+}\right)=-\frac{a}{b} W\left[-\frac{b}{a} y_{i}^{+} \exp \left(-\frac{b}{a} y_{i}^{+}+\frac{A_{h}}{a}\right)\right] .
$$

Based on the above discussion, we can see that the sign of $A_{h}$, the key parameters $\tau, \theta$ and $V_{L}$ play important roles in defining the domains of the impulsive and phase sets, and in determining the Poincaré map, as shown in Table 2. Thus, it is time to explore the existence and stability of the order-1

\begin{tabular}{|c|c|c|c|c|}
\hline \multicolumn{2}{|c|}{ Cases } & $\left(1-\frac{\delta V_{L}}{V_{L}+\beta}\right) V_{L}$ & $A_{h}$ & $\mathcal{P}\left(y_{i}^{+}\right)$ \\
\hline \multirow{6}{*}{$\left(A_{1}\right)$} & (i) & \multirow{3}{*}{$\left(1-\frac{\delta V_{L}}{V_{L}+\beta}\right) V_{L} \geq x_{1}$} & \multirow{3}{*}{$A_{h} \geq 0$} & $y_{i}^{+} \in Y_{1}$ \\
\hline & $(i i)$ & & & $y_{i}^{+} \in Y_{2}$ \\
\hline & $(\mathrm{iii})$ & & & $y_{i}^{+} \in Y_{3} \cup Y_{4}$ \\
\hline & $(i)$ & \multirow{3}{*}{$\left(1-\frac{\delta V_{L}}{V_{L}+\beta}\right) V_{L}<x_{1}$} & \multirow{3}{*}{$A_{h}<0$} & $y_{i}^{+} \in Y_{5}$ \\
\hline & $(i i)$ & & & $y_{i}^{+} \in Y_{3}^{0} \cup Y_{6}$ \\
\hline & $(\mathrm{iii})$ & & & $y_{i}^{+} \in Y_{7}$ \\
\hline \multirow{3}{*}{$\left(A_{2}\right)$} & $(i)$ & \multirow{3}{*}{$\left(1-\frac{\delta V_{L}}{V_{L}+\beta}\right) V_{L}<x^{*}$} & \multirow{3}{*}{$A_{h}<0$} & $y_{i}^{+} \in Y_{5}$ \\
\hline & (ii) & & & $y_{i}^{+} \in Y_{3}^{0} \cup Y_{6}$ \\
\hline & (iii) & & & $y_{i}^{+} \in Y_{7}$ \\
\hline
\end{tabular}
periodic solutions of model (1) and to discuss how the above vital parameters affect the dynamics of model (1).

Table 2 The signs of $A_{h}$ and domains of the Poincaré map $\mathcal{P}\left(y_{i}^{+}\right)$for all possible cases 


\section{Order-1 periodic solution for $\tau=0$}

Note that the relations between the Poincaré map $\mathcal{P}\left(y_{i}^{+}\right)$and the solutions of model (1) show that the existence and stability of the fixed point of $\mathcal{P}\left(y_{i}^{+}\right)$ indicate the existence and stability of the order-1 periodic solution or limit cycle of model (1).

The explicit expression of the Poincaré map $\mathcal{P}\left(y_{i}^{+}\right)$has been obtained in the previous section, which can be used to investigate the existence of the fixed point of $\mathcal{P}\left(y_{i}^{+}\right)$more easily, denoted by $y^{*}$, and we have $\mathcal{P}\left(y^{*}\right)=y^{*}$, i.e.

$$
y^{*}=-\frac{a}{b} W\left[-\frac{b}{a} y^{*} \exp \left(-\frac{b}{a} y^{*}+\frac{A_{h}}{a}\right)\right]+\frac{\tau}{1-\frac{\theta a}{b} W\left[-\frac{b}{a} y^{*} \exp \left(-\frac{b}{a} y^{*}+\frac{A_{h}}{a}\right)\right]} .
$$

Note that if $\tau=0$, then the above equation becomes

$$
y^{*}=-\frac{a}{b} W\left[-\frac{b}{a} y^{*} \exp \left(-\frac{b}{a} y^{*}+\frac{A_{h}}{a}\right)\right],
$$

which can be easily analyzed. To show this, we consider the following two cases:

If $\tau=0, A_{h}=0$, then the fixed point $y^{*}$ of $\mathcal{P}\left(y_{i}^{+}\right)$satisfies

$$
y^{*}=-\frac{a}{b} W\left[-\frac{b}{a} y^{*} \exp \left(-\frac{b}{a} y^{*}\right)\right],
$$

which means that any $y^{*}$ in the phase set is a fixed point of the $\mathcal{P}\left(y_{i}^{+}\right)$due to the definition of the Lambert $\mathrm{W}$ function.

If $\tau=0, A_{h} \neq 0$, then the fixed point $y^{*}$ of the $\mathcal{P}\left(y_{i}^{+}\right)$satisfies

$$
y^{*}=-\frac{a}{b} W\left[-\frac{b}{a} y^{*} \exp \left(-\frac{b}{a} y^{*}+\frac{A_{h}}{a}\right)\right],
$$

from which we can see that $\mathcal{P}\left(y^{*}\right)=y^{*}$ is true if and only if $y^{*}=0$. Therefore, $y^{*}=0$ is a unique fixed point of the $\mathcal{P}\left(y_{i}^{+}\right)$for $\tau=0, A_{h} \neq 0$.

In summary, for $\tau=0$ we have the following main results.

Theorem 4 If $\tau=0, A_{h}=0$, then the fixed point $y^{*}$ of the $\mathcal{P}\left(y_{i}^{+}\right)$is stable but not asymptotically stable. If $\tau=0, A_{h}<0$, then the boundary order-1 periodic solution $\left(x^{T}(t), 0\right)$ which corresponds with the fixed point $y^{*}=0$ is globally asymptotically stable. If $\tau=0, A_{h} \geq 0$, then the boundary order-1 periodic solution $\left(x^{T}(t), 0\right)$ is unstable.

Proof. If $\tau=0, A_{h}=0$, then it is easy to see that $y^{*}$ is a fixed point of the $\mathcal{P}\left(y_{i}^{+}\right)$and the results are follows. Note that if $\tau=0$ and $A_{h} \neq 0$, then $y^{*}=0$ is a unique fixed point of Poincaré map $\mathcal{P}\left(y_{i}^{+}\right)$, and consequently a unique boundary order-1 periodic solution of model (1) with initial condition $\left(\left(1-\frac{\delta V_{L}}{V_{L}+\beta}\right) V_{L}, 0\right)$ appears.

In fact, letting $y(t)=0$ and $\tau=0$ in system (1), yields the following subsystem:

$$
\left\{\begin{array}{l}
\frac{d x(t)}{d t}=a x(t), \quad x(t)<V_{L}, \\
x\left(t^{+}\right)=\left(1-\frac{\delta x(t)}{x(t)+\beta}\right) x(t), \quad x(t)=V_{L} .
\end{array}\right.
$$


For convenience, we denote $P_{V_{L}}=\frac{\delta V_{L}}{V_{L}+\beta}$ and $x\left(0^{+}\right)=\left(1-P_{V_{L}}\right) V_{L}$, and integrating the first equation yields

$$
x^{T}(t)=\left(1-P_{V_{L}}\right) V_{L} \exp (a t) .
$$

Letting $V_{L}=\left(1-P_{V_{L}}\right) V_{L} \exp (a T)$ and solving it with respect to period $T$, one has $T=-\frac{1}{a} \ln \left(1-P_{V_{L}}\right)$. Therefore, system (29) has a periodic solution with period $T$ as follows:

$$
\left(x^{T}(t), 0\right)=\left(\left(1-P_{V_{L}}\right) V_{L} \exp (a t), 0\right) .
$$

Next we discuss the stability of $\left(x^{T}(t), 0\right)$. To do this, for $A_{h} \neq 0$ we take any two points $q_{0}^{+}\left(\left(1-P_{V_{L}}\right) V_{L}, y_{1}^{+}\right) \in L_{3}$ and $q_{1}\left(V_{L}, y_{2}\right) \in L_{2}$ with $y_{1}^{+}, y_{2} \leq \frac{a}{b}$ from same trajectory of model (1) (Fig. 3(A) and (D)), and the both points satisfy the following equation:

$$
A_{h}=d \ln \left(1-P_{V_{L}}\right)+c P_{V_{L}} V_{L}=a \ln \frac{y_{2}}{y_{1}^{+}}-b\left(y_{2}-y_{1}^{+}\right),
$$

with $y_{2} \neq y_{1}^{+}$due to $A_{h} \neq 0$. Define $h(y)=a \ln y-b y$ with $h^{\prime}(y)=\frac{a}{y}-b$, which means that $h^{\prime}(y)>0$ for $y<\frac{a}{b}$.

Furthermore, if $A_{h}>0$, we can obtain the following relationship:

$$
a \ln \frac{y_{2}^{+}}{y_{1}^{+}}-b\left(y_{2}^{+}-y_{1}^{+}\right)>0 \text { or } a \ln \frac{y_{2}}{y_{1}}-b\left(y_{2}-y_{1}\right)>0,
$$

here $y_{1}=y_{1}^{+}$and $y_{2}=y_{2}^{+}$due to $\tau=0$. That is,

$$
a \ln \left(y_{2}^{+}\right)-b y_{2}^{+}>a \ln \left(y_{1}^{+}\right)-b y_{1}^{+} \text {or } a \ln \left(y_{2}\right)-b y_{2}>a \ln \left(y_{1}\right)-b y_{1},
$$

which indicate that $y_{2}^{+}>y_{1}^{+}$and $y_{2}>y_{1}$. Similarly, if $A_{h}<0$, then we can get $y_{2}^{+}<y_{1}^{+}$and $y_{2}<y_{1}$.

Therefore, if $\tau=0$ and $A_{h}<0$, we could assume that any solution starting from $\mathcal{N}_{5}$ experiences infinite impulsive perturbations, i.e. $y_{k}^{+} \in\left(0, Y_{i s}^{h}\right]$ for all $k \geq 0$. Based on the above analyses we can see that $y_{k}^{+}$is a monotonically decreasing series with $\lim _{k \rightarrow \infty} y_{k}^{+}=y^{*}$ and $y^{*}=0$ hold. Therefore, all those confirm that the boundary order-1 limit cycle $\left(x^{T}(t), 0\right)$ is globally attractive.

To show the local asymptotical stability of $\left(x^{T}(t), 0\right)$, we can prove it with two different methods: according to the relationship between the $T$-periodic solution and the Floquet multiplier [26, 27] or directly employing the Poincaré map $\mathcal{P}\left(y_{i}^{+}\right)$, which are given separately as follows.

Method 1: For $A_{h}<0$, we denote

$$
\begin{gathered}
P(x, y)=x(t)(a-b y(t)), \quad Q(x, y)=y(t)(c x(t)-d), \\
\alpha(x, y)=\frac{-\delta x^{2}(t)}{x(t)+\beta}, \quad \gamma(x, y)=\frac{\tau}{1+\theta y(t)}, \quad \phi(x, y)=x-V_{L}, \\
\left(x^{T}(T), y^{T}(T)\right)=\left(V_{L}, 0\right), \quad\left(x^{T}\left(T^{+}\right), y^{T}\left(T^{+}\right)\right)=\left(\left(1-P_{V_{L}}\right) V_{L}, 0\right) .
\end{gathered}
$$


Then,

$$
\begin{aligned}
& \frac{\partial P}{\partial x}=a-b y, \quad \frac{\partial Q}{\partial y}=c x-d, \quad \frac{\partial \alpha}{\partial x}=-\frac{\delta x^{2}+2 \delta \beta x}{(x+\beta)^{2}} \\
& \frac{\partial \gamma}{\partial y}=-\frac{\tau \theta}{(1+\theta y)^{2}}, \quad \frac{\partial \phi}{\partial x}=1, \quad \frac{\partial \alpha}{\partial y}=\frac{\partial \gamma}{\partial x}=\frac{\partial \phi}{\partial y}=0
\end{aligned}
$$

and

$$
\begin{aligned}
\Delta_{1} & =\frac{P_{+}\left(\frac{\partial \gamma}{\partial y} \frac{\partial \phi}{\partial x}-\frac{\partial \gamma}{\partial x} \frac{\partial \phi}{\partial y}+\frac{\partial \phi}{\partial x}\right)+Q_{+}\left(\frac{\partial \alpha}{\partial x} \frac{\partial \phi}{\partial y}-\frac{\partial \alpha}{\partial y} \frac{\partial \phi}{\partial x}+\frac{\partial \phi}{\partial y}\right)}{P \frac{\partial \phi}{\partial x}+Q \frac{\partial \phi}{\partial y}} \\
& =\frac{P_{+}\left(x^{T}\left(T^{+}\right), y^{T}\left(T^{+}\right)\right)(1-\tau \theta)}{P\left(x^{T}(T), y^{T}(T)\right)}=\frac{a\left(1-P_{V_{L}}\right) V_{L}(1-\tau \theta)}{a V_{L}} \\
& =\left(1-P_{V_{L}}\right)(1-\tau \theta) .
\end{aligned}
$$

Moreover, we have

$$
\begin{aligned}
& \exp \left(\int_{0}^{T}\left[\frac{\partial P}{\partial x}\left(x^{T}(t), y^{T}(t)\right)+\frac{\partial Q}{\partial y}\left(x^{T}(t), y^{T}(t)\right)\right] d t\right) \\
& =\exp \left(\int_{0}^{T}\left[a+c\left(1-P_{V_{L}}\right) V_{L} \exp (a t)-d\right] d t\right) \\
& =\exp \left((a-d) T+\frac{c\left(1-P_{V_{L}}\right) V_{L}}{a}(\exp (a T)-1)\right) \\
& =\exp \left(-(a-d) \frac{\ln \left(1-P_{V_{L}}\right)}{a}+\frac{c\left(1-P_{V_{L}}\right) V_{L}}{a}\left(\frac{1}{1-P_{V_{L}}}-1\right)\right) \\
& =\exp \left(\ln \frac{1}{1-P_{V_{L}}}+\frac{A_{h}}{a}\right) .
\end{aligned}
$$

Thus, the Floquet multiplier $\mu_{2}$ can be obtained as follows:

$$
\begin{aligned}
\mu_{2} & =\Delta_{1} \exp \left(\int_{0}^{T}\left[\frac{\partial P}{\partial x}\left(x^{T}(t), y^{T}(t)\right)+\frac{\partial Q}{\partial y}\left(x^{T}(t), y^{T}(t)\right)\right] d t\right) \\
& =\left(1-P_{V_{L}}\right)(1-\tau \theta) \exp \left(\ln \frac{1}{1-P_{V_{L}}}+\frac{A_{h}}{a}\right) \\
& =(1-\tau \theta) \exp \left(\frac{A_{h}}{a}\right) .
\end{aligned}
$$

Therefore, it follows from (31) that $\left|\mu_{2}\right|<1$ if $A_{h}<0$ and $\tau=0$, which indicates that the boundary order-1 periodic solution is globally stable. Meanwhile, the instability of the boundary order-1 periodic solution follows if $A_{h} \geq 0$, as shown in Fig. 3 .

Method 2: $\quad$ if $\tau=0, A_{h} \neq 0$, the Poincaré map is

$$
\mathcal{P}\left(y_{i}^{+}\right)=-\frac{a}{b} W\left[-\frac{b}{a} y_{i}^{+} \exp \left(-\frac{b}{a} y_{i}^{+}+\frac{A_{h}}{a}\right)\right],
$$

taking the derivative with respect to $y_{i}^{+}$yields

$$
\left.\frac{d \mathcal{P}\left(y_{i}^{+}\right)}{d y_{i}^{+}}\right|_{y_{i}^{+}=y^{*}}=-\frac{a}{b} \cdot \frac{W\left[-\frac{b}{a} y^{*} \exp \left(-\frac{b}{a} y^{*}+\frac{A_{h}}{a}\right)\right]}{1+W\left[-\frac{b}{a} y^{*} \exp \left(-\frac{b}{a} y^{*}+\frac{A_{h}}{a}\right)\right]}\left(\frac{1}{y^{*}}-\frac{b}{a}\right) \triangleq g\left(y^{*}\right) .
$$



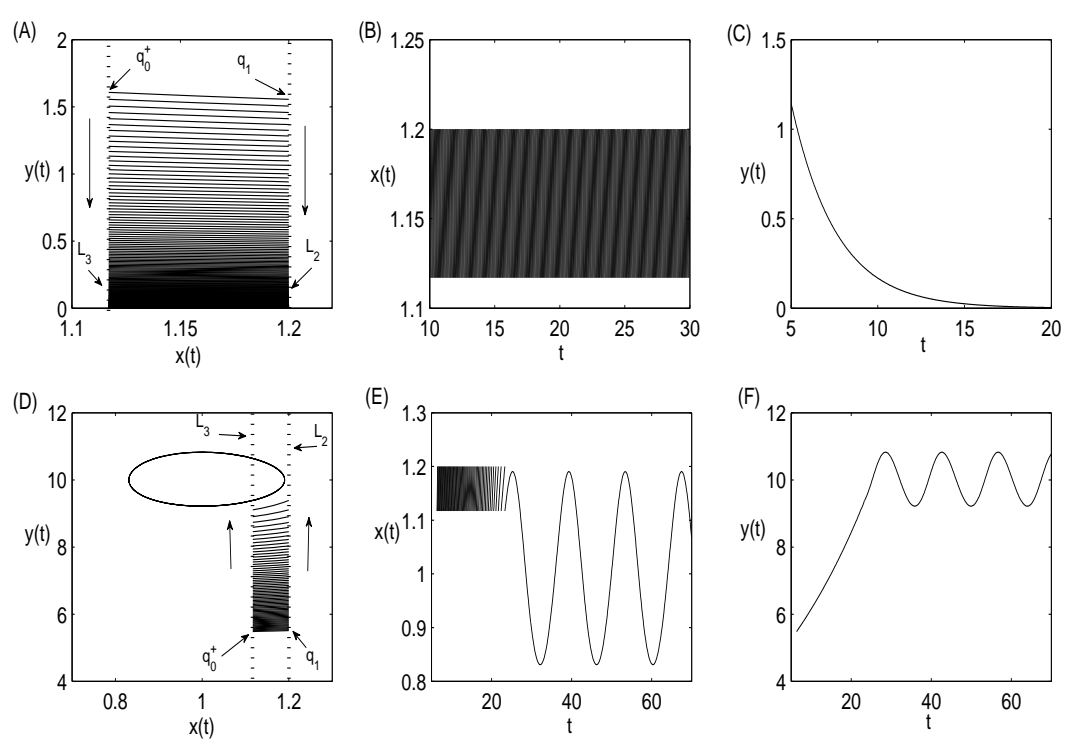

Fig. 3 Stability of boundary order-1 periodic solution $\left.\left(x^{T}(t), 0\right)\right)$. (A)-(C) Stable boundary order-1 limit cycle with $V_{L}=1.4$ and $A_{h}=-0.05, c=0.5, d=1.2 ;(\mathrm{D})$-(F) Unstable boundary order-1 limit cycle with $V_{L}=1.2$ and $A_{h}=0.003, c=0.2, d=0.2$. All other parameter values are fixed as follows: $a=1, b=0.1, \delta=0.3, \beta=4, \theta=1, \tau=0$.

The stability of the boundary order-1 periodic solution $\left(x^{T}(t), 0\right)$ is equivalent to the inequality $\left|g\left(y^{*}\right)\right|<1$ holding true. In fact, it is easy to see that the limitation

$$
\lim _{y^{*} \rightarrow 0^{+}} g\left(y^{*}\right)=e^{\frac{A_{h}}{a}},
$$

which indicates that if $A_{h}<0$, then $\left|g\left(y^{*}\right)\right|<1\left(y^{*} \rightarrow 0^{+}\right)$, and consequently the boundary order-1 periodic solution is globally stable. This completes the proof.

\section{Order-1 periodic solution for $\tau>0$}

\subsection{Some important notations and relations}

From Lemma 1 and Lemma 2 we know that if $A_{h}<0$, then the line $L_{2}$ will intersect the curve $\Gamma_{1}$ at two points $Q_{1}$ and $Q_{2}$, here $Q_{1}:\left(x_{Q_{1}}, y_{Q_{1}}\right)=$ $\left(V_{L}, Y_{I S}^{h}\right), Q_{2}:\left(x_{Q_{2}}, y_{Q_{2}}\right)=\left(V_{L}, Y_{i s}^{h}\right)$, and $\Gamma_{1}$ is tangent to $L_{3}$ at point $Q_{0}$ : $\left(x_{Q_{0}}, y_{Q_{0}}\right)=\left(\left(1-P_{V_{L}}\right) V_{L}, \frac{a}{b}\right)$. The point $Q_{2}^{+}:\left(x_{Q_{2}^{+}}, y_{Q_{2}^{+}}\right)=\left(\left(1-P_{V_{L}}\right) V_{L}, Y_{i s}^{h}+\right.$ $\left.\frac{\tau}{1+\theta Y_{i s}^{h}}\right)$ is the impulsive point of $Q_{2}$ after a single impulsive perturbation.

If $^{i s} A_{h} \geq 0$, then the curve $\Gamma_{h}$ will intersect with line $L_{3}$ at two points $P_{1}$ and $P_{2}$, and tangent to the line $x=V_{L}$ at point $T:\left(x_{T}, y_{T}\right)=\left(V_{L}, \frac{a}{b}\right)$, here $P_{1}:\left(x_{P_{1}}, y_{P_{1}}\right)=\left(\left(1-P_{V_{L}}\right) V_{L}, Y_{\text {max }}^{h}\right), P_{2}:\left(x_{P_{2}}, y_{P_{2}}\right)=\left(\left(1-P_{V_{L}}\right) V_{L}, Y_{m i n}^{h}\right)$, 
the point $T$ maps to the point $T^{+}:\left(x_{T^{+}}, y_{T+}\right)=\left(\left(1-P_{V_{L}}\right) V_{L}, \frac{a}{b}+\frac{\tau}{1+\theta \frac{a}{b}}\right)$ after a single impulsive effect.

We define the useful point $Q:\left(x_{Q}, y_{Q}\right)=\left(V_{L}, \frac{\sqrt{\tau \theta}-1}{\theta}\right)$, and if the point $Q$ is located in the impulsive set, it will map to the impulsive point $Q^{+}$: $\left(x_{Q^{+}}, y_{Q^{+}}\right)=\left(\left(1-P_{V_{L}}\right) V_{L}, \frac{2 \sqrt{\tau \theta}-1}{\theta}\right)$ after a single impulsive effect.

Lemma 5 Denote $q_{i}\left(V_{L}, y_{i}\right)$ to be a point which is located in the impulsive set, and $q_{i}^{+}\left(\left(1-P_{V_{L}}\right) V_{L}, y_{i}^{+}\right)$to be the impulsive point of $q_{i}$. Then we can define the line segment joining the two points as $\overline{q_{i} q_{i}^{+}}(i=1,2,3, \cdots)$, and the slope of line segments $K_{\overline{q_{i} q_{i}^{+}}}$satisfy the following relation:

$$
K \overline{q_{i+1} q_{i+1}^{+}}>K_{\overline{q_{i} q_{i}^{+}}} \text {if } y_{i+1}>y_{i} .
$$

Proof. It follows from model (1) that $y_{i}^{+}=y_{i}+\frac{\tau}{1+\theta y_{i}}$, which indicates that the line segments $\overline{q_{i} q_{i}^{+}}(i=1,2,3, \cdots)$ are not parallel to each other. Thus, if $\tau>0$, then the slope of line $\overline{q_{i} q_{i}^{+}}$is $K \frac{}{q_{i} q_{i}^{+}}=-\frac{\tau}{P_{V_{L}} V_{L}\left(1+\theta y_{i}\right)}<0$, and by simple calculation we have $\frac{d K \overline{q_{i} q_{i}^{+}}}{d y_{i}}=\frac{\tau \theta}{P_{V_{L}} V_{L}\left(1+\theta y_{i}\right)^{2}}>0$. Therefore, the slope $K_{\overline{q_{i} q_{i}^{+}}}$is a monotonically increasing function of $y_{i}$. This means that for any two points $q_{i}\left(V_{L}, y_{i}\right), q_{i+1}\left(V_{L}, y_{i+1}\right)$ in the impulsive set, the slope of the impulsive line segments satisfies the relation $K_{q_{i+1} q_{i+1}^{+}}>K_{q_{i} q_{i}^{+}}$if $y_{i+1}>y_{i}$.

Note that the impulsive segments $\overline{q_{i} q_{i}^{+}}(i=1,2, \cdots)$ in Lemma 5 are not parallel which is caused by the nonlinear pulse. Therefore, from a qualitative point of view, it will result in some difficulties for analyzing the dynamics when nonlinear impulsive functions are considered, and the dynamic behavior of system (1) would be richer [22].

Lemma 6 If $A_{h} \geq 0$ and $\tau>0$, then the Poincaré map satisfies the following inequality

$$
\mathcal{P}\left(y_{*}\right)>y_{*} \text {, for all } y_{*} \in\left(0, Y_{\text {min }}^{h}\right) \subset\left(0, \frac{a}{b}\right) .
$$

Proof. Note that any solution $(x(t), y(t))$ of system (2) with initial value $\left(x_{0}, y_{0}\right)$ satisfies equation (4): $a \ln y-b y+d \ln x-c x=h_{0}$ with $h_{0}=a \ln y_{0}-$ $b y_{0}-c x_{0}+d \ln x_{0}$. Thus, there is a trajectory which crosses the point $q_{0}((1-$ $\left.\left.P_{V_{L}}\right) V_{L}, y_{*}\right)$ and intersects with $L_{2}$ at point $q_{1}\left(V_{L}, y_{* *}\right)$ under the line $L_{1}$ (i.e. $\left.y=\frac{a}{b}\right)$, here we assume that $0<y_{*}<\frac{a}{b}$. Moreover, it is easy to obtain the following relationship:

$$
a \ln y_{* *}-b y_{* *}+d \ln V_{L}-c V_{L}=a \ln y_{*}-b y_{*}-c\left(1-P_{V_{L}}\right) V_{L}+d \ln \left(1-P_{V_{L}}\right) V_{L},
$$

i.e. we have

$$
\left(-\frac{b}{a} y_{* *}\right) \exp \left(-\frac{b}{a} y_{* *}\right)=-\frac{b}{a} y_{*} \exp \left(-\frac{b}{a} y_{*}+\frac{A_{h}}{a}\right) .
$$


It follows from (33) that if $A_{h} \geq 0$, then we have

$$
\left(-\frac{b}{a} y_{* *}\right) \exp \left(-\frac{b}{a} y_{* *}\right) \leq-\frac{b}{a} y_{*} \exp \left(-\frac{b}{a} y_{*}\right) .
$$

Considering the function $D(x)=-x \exp (-x)$, by calculating we can see that $D^{\prime}(x)<0$ if $0<x<1$ and $D^{\prime}(x)>0$ if $x>1$. It follows from $\frac{b}{a} y_{* *}, \frac{b}{a} y_{*} \in$ $(0,1)$ that we have $y_{* *} \geq y_{*}$. Further, according to $y_{* *}^{+}=y_{* *}+\frac{\tau}{1+\theta y_{* *}}$ and $\mathcal{P}\left(y_{*}\right)=y_{* *}^{+}$, we obtain the following inequality:

$$
\mathcal{P}\left(y_{*}\right)>y_{*}, \quad y_{*} \in\left(0, Y_{\text {min }}^{h}\right) \subset\left(0, \frac{a}{b}\right), A_{h} \geq 0, \tau>0 .
$$

5.2 Existence and stability of order-1 periodic solution

In order to discuss the existence and stability of the fixed point of the Poincaré map $\mathcal{P}\left(y_{i}^{+}\right)$for $\tau>0$, we first analyze its properties.

Theorem 7 a) The monotonicities of Poincaré map $\mathcal{P}\left(y_{i}^{+}\right)$for cases $\left(A_{1}\right)$ and $\left(A_{2}\right)$ listed in Table 2 are as follows:

$\left(A_{11}\right) \quad V_{L}>x^{*}, x_{1} \leq\left(1-P_{V_{L}}\right) V_{L}$ and $A_{h} \geq 0$.

$(i)$ : The Poincaré map is increasing on $\left[0, y_{P_{2}}\right]$, and decreasing on $\left[y_{P_{1}},+\infty\right)$ for $\frac{\sqrt{\tau \theta}-1}{\theta} \leq 0$.

(ii): The Poincaré map is increasing on $\left[y_{P_{1}},+\infty\right)$, and decreasing on $\left[0, y_{P_{2}}\right]$ for $\frac{\sqrt{\tau \theta}-1}{\theta} \geq \frac{a}{b}$.

(iii): The Poincaré map is decreasing on $\left[0, y_{M_{2}}\right]$ and $\left[y_{P_{1}}, y_{M_{1}}\right]$, and increasing on $\left[y_{M_{2}}, y_{P_{2}}\right]$ and $\left[y_{M_{1}},+\infty\right)$ for $0<\frac{\sqrt{\tau \theta}-1}{\theta}<\frac{a}{b}$, where $y_{M_{2}}=$ $\min \left\{y^{+}: \mathcal{P}\left(y^{+}\right)=y_{Q^{+}}\right\}, y_{M_{1}}=\max \left\{y^{+}: \mathcal{P}\left(y^{+}\right)=y_{Q^{+}}\right\}$.

$\left(A_{12}\right) \quad V_{L}>x^{*},\left(1-P_{V_{L}}\right) V_{L}<x_{1}$ and $A_{h}<0$.

$(i)$ : The Poincaré map is increasing on $\left[0, y_{Q_{0}}\right]$, and decreasing on $\left[y_{Q_{0}},+\infty\right)$ for $\frac{\sqrt{\tau \theta}-1}{\theta} \leq 0$.

(ii): The Poincaré map is decreasing on $\left[0, y_{m_{2}}\right]$ and $\left[y_{Q_{0}}, y_{m_{1}}\right]$, and increasing on $\left[y_{m_{2}}, y_{Q_{0}}\right]$ and $\left[y_{m_{1}},+\infty\right)$ for $0<\frac{\sqrt{\tau \theta}-1}{\theta}<Y_{i s}^{h}$, where $y_{m_{2}}=$ $\min \left\{y^{+}: \mathcal{P}\left(y^{+}\right)=y_{Q^{+}}\right\}, y_{m_{1}}=\max \left\{y^{+}: \mathcal{P}\left(y^{+}\right)=y_{Q^{+}}\right\}$.

(iii): The Poincaré map is increasing on $\left[y_{Q_{0}},+\infty\right)$, and decreasing on $\left[0, y_{Q_{0}}\right]$ for $\frac{\sqrt{\tau \theta}-1}{\theta} \geq Y_{i s}^{h}$.

$\left(A_{2}\right): V_{L} \leq x^{*}$ and $A_{h}<0$.

$(i)$ : The Poincaré map is increasing on $\left[0, y_{Q_{0}}\right]$, and decreasing on $\left[y_{Q_{0}},+\infty\right)$ for $\frac{\sqrt{\tau \theta}-1}{\theta} \leq 0$.

(ii): The Poincaré map is decreasing on $\left[0, y_{m_{2}}\right]$ and $\left[y_{Q_{0}}, y_{m_{1}}\right]$, and increasing on $\left[y_{m_{2}}, y_{Q_{0}}\right]$ and $\left[y_{m_{1}},+\infty\right)$ for $0<\frac{\sqrt{\tau \theta}-1}{\theta}<Y_{i s}^{h}$, where $y_{m_{2}}=$ $\min \left\{y^{+}: \mathcal{P}\left(y^{+}\right)=y_{Q^{+}}\right\}, y_{m_{1}}=\max \left\{y^{+}: \mathcal{P}\left(y^{+}\right)=y_{Q^{+}}\right\}$. 
(iii): The Poincaré map is increasing on $\left[y_{Q_{0}},+\infty\right)$, and decreasing on $\left[0, y_{Q_{0}}\right]$ for $\frac{\sqrt{\tau \theta}-1}{\theta} \geq Y_{i s}^{h}$.

b) There exists a horizontal asymptote $y=\tau$ for $\mathcal{P}\left(y_{i}^{+}\right)$as $y_{i}^{+} \rightarrow+\infty$.

Proof. $a)$ Assuming that the point $q_{i}^{+}=\left(\left(1-P_{V_{L}}\right) V_{L}, y_{i}^{+}\right)$belongs to the phase set, the solution of model (1) starting from $q_{i}^{+}$intersects with the line $x=V_{L}$ at a point $q_{i+1}=\left(V_{L}, y_{i+1}\right)$. Note that $y_{i+1}$ can be determined by $y_{i}^{+}$ due to the both point are in one trajectory $\Gamma$, denoted by $y_{i+1}=f\left(y_{i}^{+}\right)$.

It follows from the vector field of system (1) that the domain of Poincaré map $\mathcal{P}\left(y_{i}^{+}\right)$in cases $\left(A_{1}\right)\left(A_{11}\right)$ is $\left[0, y_{P_{2}}\right] \cup\left[y_{P_{1}},+\infty\right)$. For all subcases $\left(A_{11}\right)(i)$, (ii), (iii), based on the uniqueness of solution of model (2), the function $f$ is increasing on $\left[0, y_{P_{2}}\right]$ and decreasing on $\left[y_{P_{1}},+\infty\right)$.

Moreover, for case $\left(A_{11}\right)(i)$ the impulsive function $G$ is increasing on $\left[0, y_{T}\right]$. Thus, according to the definition of $\mathcal{P}\left(y_{i}^{+}\right)$, we know that it is increasing on $\left[0, y_{P_{2}}\right]$ and decreasing on $\left[y_{P_{1}},+\infty\right)$. For case $\left(A_{11}\right)(i i)$, the impulsive function $G$ is decreasing on $\left[0, y_{T}\right]$, which indicates that $\mathcal{P}\left(y_{i}^{+}\right)$is decreasing on $\left[0, y_{P_{2}}\right]$ and increasing on $\left[y_{P_{1}},+\infty\right)$. For case $\left(A_{11}\right)(i i i)$, impulsive function $G$ is decreasing on $\left[0, y_{Q}\right]$, and increasing on $\left[y_{Q}, y_{T}\right]$. Thus, $\mathcal{P}\left(y_{i}^{+}\right)$is decreasing on $\left[0, y_{M_{2}}\right]$ and $\left[y_{P_{1}}, y_{M_{1}}\right]$, and increasing on $\left[y_{M_{2}}, y_{P_{2}}\right]$ and $\left[y_{M_{1}},+\infty\right)$.

By using the same methods we can prove that the cases $\left(A_{12}\right)(i),(i i),(i i i)$ and cases $\left(A_{2}\right)(i),(i i),(i i i)$ in Theorem 7 are true.

b) It follows from the Lambert $\mathrm{W}$ function that

$$
\lim _{y_{i}^{+} \rightarrow+\infty}-\frac{a}{b} W\left[-\frac{b}{a} y_{i}^{+} \exp \left(-\frac{b}{a} y_{i}^{+}+\frac{A_{h}}{a}\right)\right]=0 .
$$

It follows from equality (25) that we can calculate the limitation about $\mathcal{P}\left(y_{i}^{+}\right)$ as follows:

$$
\lim _{y_{i}^{+} \rightarrow+\infty} \mathcal{P}\left(y_{i}^{+}\right)=\tau,
$$

which indicates that there exists a horizontal asymptote $y=\tau$ for $\mathcal{P}\left(y_{i}^{+}\right)$as $y_{i}^{+} \rightarrow+\infty$. This completes the proof.

Based on the above properties of the Poincaré map, we have the following main results related to the existence of the fixed point of the $\mathcal{P}\left(y_{i}^{+}\right)$for $\tau>0$.

Theorem 8 For case $\left(A_{11}\right)(i)$ (or $\left.(i i)\right)$, if $y_{T^{+}}>y_{P_{1}}$ then the Poincaré map $\mathcal{P}\left(y_{i}^{+}\right)$exists with at least a fixed point, and consequently for system (1) there exists an order-1 periodic solution. If $y_{T^{+}}<y_{P_{1}}$, then $\mathcal{P}\left(y_{i}^{+}\right)$does not have any fixed point.

Proof. According to the results shown in subsection 5.1 we know that in case $\left(A_{11}\right)$, there is a curve $\Gamma_{h}$ which intersects with line $L_{3}$ at two points $P_{1}$ and $P_{2}$, and is tangent to the line $L_{2}$ at point $T\left(V_{L}, \frac{a}{b}\right)$. Obviously, if $y_{T^{+}}=y_{P_{1}}$, then the curve $\widehat{P_{1} T}$ is an order-1 periodic solution for system (1). 
For case $\left(A_{11}\right)(i)$, if $y_{T^{+}}>y_{P_{1}}$, then it follows that $\mathcal{P}\left(y_{p_{1}}\right)=y_{T^{+}}$that the point $T^{+}$lies above the point $P_{1}$, and we have

$$
\mathcal{P}\left(y_{P_{1}}\right)>y_{P_{1}}
$$

Moreover, the solution with initial value $T^{+}$meets the line $L_{2}$ at a point $T_{1}$ which lies below the point $T$, i.e. $y_{T_{1}}<y_{T}$. Due to the impulsive function $G$ is increasing on $\left[0, y_{T}\right]$ in this case, we have $G\left(y_{T_{1}}\right)<G\left(y_{T}\right)$, i.e. $y_{T_{1}}^{+}<y_{T}^{+}$. All these results confirm that for case $\left(A_{11}\right)(i)$ the Poincaré map satisfies the relationship

$$
\mathcal{P}\left(y_{T^{+}}\right)<y_{T^{+}} .
$$

It follows from the inequalities (36) and (37) that one fixed point exits in the interval $\left(y_{P_{1}}, y_{T^{+}}\right)$, which corresponds to an order-1 periodic solution for system (1).

If $y_{T^{+}}<y_{P_{1}}$, then any solution of system (1) will map to the interval $\left[\tau, y_{T^{+}}\right]$after a single impulsive effect in this case. Thus, if $\tau \leq y_{P_{2}}$, based on the inequality (34) in Lemma 6, then the trajectory initiating from $y^{+}$ with $\tau \leq y^{+} \leq y_{P_{2}}$ will arrive at $L_{2}$ and experience a finite number of pulses and eventually tends to the interior of closed trajectory $\Gamma_{h}$ (here we denote its interior as $\operatorname{Int} \Omega_{h}$ ), and will be free from any more impulsive effects. If $y_{P_{2}}<\tau<y_{T^{+}}$, obviously, any solution of system (1) will map to the $\operatorname{Int} \Omega_{h}$ after a single impulsive effect. In conclusion there does not exist any fixed point for $y_{T^{+}}<y_{P_{1}}$.

For case $\left(A_{11}\right)(i i)$, if $y_{T^{+}}>y_{P_{1}}$, on the one hand, inequality (36) $\mathcal{P}\left(y_{P_{1}}\right)>$ $y_{P_{1}}$ holds true. On the other hand, the impulsive function $G$ is decreasing on $\left[0, y_{T}\right]$ for this case, so the solution initiating from $y_{i}^{+}$with $y_{i}^{+} \in\left[0, y_{P_{2}}\right] \cup$ $\left[y_{P_{1}},+\infty\right)$ will map to the interval $\left[y_{T^{+}}, \tau\right]$ after a single impulsive effect. Then, the trajectory initiating from the point $P_{\tau}\left(\left(1-P_{V_{L}}\right) V_{L}, \tau\right)$ will satisfy the inequality

$$
\mathcal{P}(\tau)<\tau \text {. }
$$

It follows from the inequalities (36) and (38) that there exists a fixed point in the interval $\left(y_{P_{1}}, \tau\right)$.

If $y_{T^{+}}<y_{P_{1}}$, any solution of system (1) will map to interval $\left[y_{T^{+}}, \tau\right]$ after a single impulsive effect for this case. If $\tau>y_{P_{1}}$, in terms of $\mathcal{P}\left(y_{P_{1}}\right)=y_{T^{+}}<y_{P_{1}}$ and the inequality (34) in Lemma 6 , we conclude that any solution initiating from the point $\left(\left(1-P_{V_{L}}\right) V_{L}, y^{+}\right)$with $y^{+} \in\left(y_{P_{1}}, \tau\right)$ experiences a finite number of pulses and then enters into Int $\Omega_{h}$, which indicates that no fixed point exists in the interval $\left[y_{P_{1}}, \tau\right]$ at all. If $\tau \leq y_{P_{1}}$, any solution of system (1) experiences a single pulse and then enters into Int $\Omega_{h}$, and it will also be free from any impulsive effects after that. So, no fixed point exists for this situation. This completes the proof.

Theorem 9 For case $\left(A_{11}\right)($ iii $)$, if $y_{T^{+}}>y_{P_{1}}$, the Poincaré map $\mathcal{P}\left(y_{i}^{+}\right)$exists with a fixed point. If $y_{T^{+}}<y_{P_{1}}$, no fixed point exists for the Poincaré map $\mathcal{P}\left(y_{i}^{+}\right)$. 
Proof. Obviously, if $y_{T^{+}}=y_{P_{1}}$, the curve $\widehat{P_{1} T}$ is an order-1 periodic solution of system (1). Otherwise, we consider two cases:

$$
\text { (1) } y_{T^{+}} \geq \tau \text {, and (2) } y_{T^{+}}<\tau \text {. }
$$

For case (1): If $y_{T^{+}}>y_{P_{1}}$, then we have $\mathcal{P}\left(y_{P_{1}}\right)>y_{P_{1}}$. Furthermore, based on the exact domain of $\mathcal{P}\left(y_{i}^{+}\right)$that we discussed in the previous section, any impulsive point lies below the point $T^{+}$for $y_{T^{+}} \geq \tau$. Therefore, the inequality $\mathcal{P}\left(y_{T^{+}}\right)<y_{T^{+}}$is true, which indicates that there exists a fixed point in the interval $\left(y_{P_{1}}, y_{T^{+}}\right)$for the Poincaré map.

If $y_{T^{+}}<y_{P_{1}}$, by employing similar methods as shown in proof of Theorem 8 , it is easy to know that any solution of system (1) experiences a finite number of pulse effects and then enters into Int $\Omega_{h}$, when it is then free from impulsive effects.

For case (2): If $y_{T^{+}}>y_{P_{1}}$, on the one hand, $\mathcal{P}\left(y_{P_{1}}\right)>y_{P_{1}}$ holds true. On the other hand, the point $P_{\tau}\left(\left(1-P_{V_{L}}\right) V_{L}, \tau\right)$ is the highest impulsive point due to $y_{T^{+}}<\tau$. Thus, we have $\mathcal{P}(\tau)<\tau$, and the theorem is true under this circumstance.

If $y_{T^{+}}<y_{P_{1}}$, any solution of system (1) experiences a finite number of pulse effects and then enters into $\operatorname{Int} \Omega_{h}$.

Theorem 10 For case $\left(A_{12}\right)(i)($ or $($ iii $))$, the Poincaré map $\mathcal{P}\left(y_{i}^{+}\right)$exists with at least a fixed point and there exists at least an order-1 periodic solution for system (1).

Proof. In case $\left(A_{12}\right)$, a curve $\Gamma_{1}$ is tangent to $L_{3}$ at point $Q_{0}\left(\left(1-P_{V_{L}}\right) V_{L}, \frac{a}{b}\right)$ and intersects with the line $L_{2}$ at two points $Q_{1}$ and $Q_{2}$. If $\mathcal{P}\left(y_{Q_{0}}\right)=y_{Q_{2}^{+}}=$ $y_{Q_{0}}=\frac{a}{b}$, obviously the curve $\widehat{Q_{0} Q_{2}}$ is an order-1 periodic solution of system (1).

For case $\left(A_{12}\right)(i)$, if $y_{Q_{2}^{+}}>y_{Q_{0}}$ or $y_{Q_{2}^{+}}<y_{Q_{0}}$, the trajectory initiating from the point $Q_{2}^{+}$intersects with the line $L_{2}$ at a point denoted by $Q_{3}\left(V_{L}, y_{Q_{3}}\right)$, here $y_{Q_{3}}<y_{Q_{2}}$, then the point $Q_{3}$ maps to a point $Q_{3}^{+}\left(\left(1-P_{V_{L}}\right) V_{L}, y_{Q_{3}^{+}}\right)$, and $y_{Q_{3}^{+}}=G\left(y_{Q_{3}}\right)$. The impulsive function $G$ is increasing on $\left[0, y_{Q_{2}}\right]$ for case $\left(A_{12}\right)(i)$. Therefore, the Poincaré map $\mathcal{P}\left(y_{Q_{2}^{+}}\right)$satisfies the following relationship

$$
\mathcal{P}\left(y_{Q_{2}^{+}}\right)<y_{Q_{2}^{+}} \cdot
$$

On the other hand, the point $P_{\tau}$ is the lowest impulsive point of the exact phase set here, then we must have

$$
\mathcal{P}(\tau)>\tau
$$

Based on the inequalities (39) and (40) we conclude that there exists at least one fixed point for the $\mathcal{P}\left(y_{i}^{+}\right)$, i.e. system (1) has at least an order-1 periodic solution. 
For case $\left(A_{12}\right)(i i i)$, the impulsive function $G$ is decreasing on $\left[0, y_{Q_{2}}\right]$. If $y_{Q_{2}^{+}}>y_{Q_{0}}$ or $y_{Q_{2}^{+}}<y_{Q_{0}}$, we have the following inequality

$$
\mathcal{P}\left(y_{Q_{2}^{+}}\right)>y_{Q_{2}^{+}},
$$

besides, the point $P_{\tau}\left(\left(1-P_{V_{L}}\right) V_{L}, \tau\right)$ is the highest impulsive point, and inequality (38) $\mathcal{P}(\tau)<\tau$ holds true. Therefore, there exists at least one fixed point for the $\mathcal{P}\left(y_{i}^{+}\right)$, i.e. system (1) has at least an order-1 periodic solution.

Theorem 11 For case $\left(A_{12}\right)(i i)$, the Poincaré map exists with at least one fixed point, i.e. system (1) has at least an order-1 periodic solution.

Proof. Obviously, if $y_{Q_{2}^{+}}=y_{Q_{0}}$, the curve $\widehat{Q_{0} Q_{2}}$ should be an order-1 periodic solution of system (1). Otherwise, we consider two cases:

$$
\text { (1) } y_{Q_{2}^{+}} \geq \tau \text {, and (2) } y_{Q_{2}^{+}}<\tau \text {. }
$$

For case (1): If $y_{Q_{2}^{+}}>y_{Q_{0}}$, we obtain

$$
\mathcal{P}\left(y_{Q_{0}}\right)>y_{Q_{0}} .
$$

Besides, the point $Q_{2}^{+}$is the highest impulsive point, then the inequality $\mathcal{P}\left(y_{Q_{2}^{+}}\right)<y_{Q_{2}^{+}}$holds true. Thus, according to these two inequalities above, there exists a fixed point for the Poincaré map $\mathcal{P}\left(y_{i}^{+}\right)$.

If $y_{Q_{2}^{+}}<y_{Q_{0}}$, then we have

$$
\mathcal{P}\left(y_{Q_{0}}\right)<y_{Q_{0}} .
$$

Moreover, the point $Q^{+}$is the lowest impulsive point, then we obtain an inequality as follows:

$$
\mathcal{P}\left(y_{Q^{+}}\right) \geq y_{Q^{+}},
$$

which shows that there exists a fixed point for the Poincaré map $\mathcal{P}\left(y_{i}^{+}\right)$.

For case (2): If $y_{Q_{2}^{+}}>y_{Q_{0}}$, on the one hand, $\mathcal{P}\left(y_{Q_{0}}\right)>y_{Q_{0}}$ is true obviously. On the other hand, if the point $P_{\tau}\left(\left(1-P_{V_{L}}\right) V_{L}, \tau\right)$ is the highest impulsive point, then $\mathcal{P}(\tau)<\tau$ holds true. With these two inequalities we can clarify that the Poincaré map $\mathcal{P}\left(y_{i}^{+}\right)$has a fixed point.

If $y_{Q_{2}^{+}}<y_{Q_{0}}$, then $\mathcal{P}\left(y_{Q_{0}}\right)<y_{Q_{0}}$. Furthermore, as we know that the point $Q^{+}$is the lowest impulsive point in this case, we obtain the inequality $\mathcal{P}\left(y_{Q^{+}}\right) \geq y_{Q^{+}}$. It shows that the Poincaré map $\mathcal{P}\left(y_{i}^{+}\right)$exists a fixed point, i.e. system (1) has an order-1 periodic solution. This completes the proof.

From Table 1 and Theorem 3 we know that the exact domains of the impulsive and phase sets and the formula of the Poincaré map for case $\left(A_{2}\right)$ are exactly the same as $\left(A_{12}\right)$. Therefore, for case $\left(A_{2}\right)(i),(i i),(i i i)$, we have the same conclusions corresponding to the case $\left(A_{12}\right)(i),(i i),(i i i)$. 
Theorem 12 For case $\left(A_{2}\right)(i),(i i),($ iii $)$, the fixed point of the Poincaré map $\mathcal{P}\left(y_{i}^{+}\right)$exists and consequently system (1) exists an order-1 periodic solution.

Based on the monotonicity of Poincaré map $\mathcal{P}\left(y_{i}^{+}\right)$in Theorem 7 and the existence of the fixed point of the $\mathcal{P}\left(y_{i}^{+}\right)$that we have discussed above, we will consider the stability of the order- 1 periodic solution of system (1) in the following theorems.

Theorem 13 For case $\left(A_{11}\right)(i)$, if $\mathcal{P}\left(y_{P_{1}}\right)>y_{P_{1}}$, then the unique positive fixed point $y^{*}$ of $\mathcal{P}\left(y_{i}^{+}\right)$is globally stable provided that $\tau \geq y_{P_{1}}$ and $\mathcal{P}^{2}\left(y_{i}^{+}\right)>$ $y_{i}^{+}$for all $y_{i}^{+} \in\left[y_{P_{1}}, y^{*}\right)$.

Proof. For case $\left(A_{11}\right)(i)$, it follows from Theorem 8 that if $\mathcal{P}\left(y_{P_{2}}\right)=\mathcal{P}\left(y_{P_{1}}\right)>$ $y_{P_{1}}$, then the $\mathcal{P}\left(y_{i}^{+}\right)$exists one fixed point. Moreover, according to $A_{h} \geq 0$ for case $\left(A_{1}\right)$ and Lemma 6 we know that $\mathcal{P}\left(y_{i}^{+}\right)>y_{i}^{+}$for all $y_{i}^{+} \in\left(0, y_{P_{2}}\right)$. Besides, it is easy to see that $\mathcal{P}(0)=\tau>0$, then the fixed point does not belong to the interval $\left[0, y_{P_{2}}\right]$. Furthermore, since $\mathcal{P}\left(y_{i}^{+}\right)$is decreasing on $\left[y_{P_{1}},+\infty\right]$, the uniqueness of fixed point for $\mathcal{P}\left(y_{i}^{+}\right)$on $\left[y_{P_{1}},+\infty\right)$ follows.

If $y_{P_{1}} \leq y_{i}^{+}<y^{*}$, it follows from monotonicity of $\mathcal{P}\left(y_{i}^{+}\right)$on the interval $\left[y_{P_{1}}, y^{*}\right)$ that we have $\mathcal{P}\left(y_{P_{1}}\right) \geq \mathcal{P}\left(y_{i}^{+}\right)>y^{*}$. Further, according to the condition $\mathcal{P}^{2}\left(y_{i}^{+}\right)>y_{i}^{+}$for all $y_{i}^{+} \in\left[y_{P_{1}}, y^{*}\right)$ we have $y_{i}^{+}<\mathcal{P}^{2}\left(y_{i}^{+}\right)<y^{*}$. By induction, we conclude that $\mathcal{P}^{2(j-1)}\left(y_{i}^{+}\right)<\mathcal{P}^{2 j}\left(y_{i}^{+}\right)<y^{*}$ for all $j \geq 1$, which means that $\mathcal{P}^{2 j}\left(y_{i}^{+}\right)$is monotonically increasing, and $\lim _{j \rightarrow+\infty} \mathcal{P}^{2 j}\left(y_{i}^{+}\right)=y^{*}, y_{i}^{+} \in$ $\left[y_{P_{1}}, y^{*}\right)$.

If $\tau \geq y_{P_{1}}$, based on the monotonicity of $\mathcal{P}\left(y_{i}^{+}\right)$, there exists a positive integer $l$ such that $\mathcal{P}^{l}\left(y_{i}^{+}\right) \in\left[y_{P_{1}}, y^{*}\right]$ for any $y_{i}^{+} \in\left[0, y_{P_{2}}\right] \cup\left(y^{*},+\infty\right)$. Then, for any $y_{i}^{+} \in\left[0, y_{P_{2}}\right] \cup\left(y^{*},+\infty\right)$ we have $\lim _{j \rightarrow+\infty} \mathcal{P}^{l+2 j}\left(y_{i}^{+}\right)=y^{*}$ monotonically. Therefore, the results shown in Theorem 13 are true, and the proof is completed.

Theorem 14 For case $\left(A_{11}\right)($ ii $)$, if $\mathcal{P}\left(y_{P_{1}}\right)>y_{P_{1}}$, then the fixed point of the $\mathcal{P}\left(y_{i}^{+}\right)$is globally stable provided that it is unique.

Proof. Based on Theorem 8, if $\mathcal{P}\left(y_{P_{2}}\right)=\mathcal{P}\left(y_{P_{1}}\right)>y_{P_{1}}$, then the fixed point of the $\mathcal{P}\left(y_{i}^{+}\right)$exists. By using the same analytical methods as those shown in Theorem 13, no fixed point in $\left[0, y_{P_{2}}\right]$ exists, and the fixed point must be located in the interval $\left(y_{P_{1}},+\infty\right)$. Furthermore, we assume that it is unique.

For case $\left(A_{11}\right)(i i)$, the Poincaré map $\mathcal{P}\left(y_{i}^{+}\right)$is decreasing on $\left[0, y_{P_{2}}\right]$ and increasing on $\left[y_{P_{1}},+\infty\right)$. If the fixed point $y^{*} \in\left(y_{P_{1}},+\infty\right)$ is unique, then for any $y_{i}^{+} \in\left[y_{P_{1}}, y^{*}\right)$, according to $y_{i}^{+}<\mathcal{P}\left(y_{i}^{+}\right)<y^{*}$, we know that $\mathcal{P}^{j}\left(y^{+}\right)$is monotonically increasing as $j$ increases, and $\lim _{j \rightarrow+\infty} \mathcal{P}^{j}\left(y_{i}^{+}\right)=y^{*}$. For any $y_{i}^{+} \in\left(y^{*},+\infty\right)$, we have $y^{*}<\mathcal{P}\left(y_{i}^{+}\right)<y_{i}^{+}$, based on the monotonicity of $\mathcal{P}\left(y_{i}^{+}\right)$, we conclude that $\mathcal{P}^{j}\left(y_{i}^{+}\right)$is monotonically decreasing as $j$ increases, which means that $\lim _{j \rightarrow+\infty} \mathcal{P}^{j}\left(y_{i}^{+}\right)=y^{*}$.

For any $y_{i}^{+} \in\left[0, y_{P_{2}}\right]$, there must be $\mathcal{P}\left(y_{i}^{+}\right) \in\left[y_{P_{1}},+\infty\right)$. Therefore, we have $\lim _{j \rightarrow+\infty} \mathcal{P}^{1+j}\left(y_{i}^{+}\right)=y^{*}$ for all $y_{i}^{+} \in\left[0, y_{P_{2}}\right]$. In conclusion, the result shown in Theorem 14 is true, which completes the proof. 
Theorem 15 For case $\left(A_{11}\right)($ iii $)$, if $\mathcal{P}\left(y_{P_{1}}\right)>y_{P_{1}}$ and the fixed point is $u$ nique for the Poincaré map $\mathcal{P}\left(y_{i}^{+}\right)$, then we have the following results:

a) If $\mathcal{P}\left(y_{M_{i}}\right)>y_{M_{i}}(i=1,2)$, then the unique fixed point $y^{*}$ is globally stable.

b) If $y_{P_{1}} \leq \mathcal{P}\left(y_{M_{2}}\right)=\mathcal{P}\left(y_{M_{1}}\right) \leq y_{M_{1}}$, then the fixed point $y^{*}$ is globally stable provided that $\mathcal{P}^{2}\left(y_{i}^{+}\right)>y_{i}^{+}$for $y_{i}^{+} \in\left[y_{P_{1}}, y^{*}\right)$.

Proof. It follows from Lemma 6 and Theorem 9 that there exists at least one fixed point $y^{*}$, which is located in the interval $\left[y_{P_{1}},+\infty\right)$ rather than in $\left[0, y_{P_{2}}\right]$. If we further assume that the fixed point of the $\mathcal{P}\left(y_{i}^{+}\right)$is unique, we can show the global stability as follows.

a) If $\mathcal{P}\left(y_{M_{i}}\right)>y_{M_{i}}(i=1,2)$, then the unique fixed point $y^{*}$ is located in the interval $\left[y_{M_{1}},+\infty\right)$. According to Theorem 7 we know that the Poincaré map $\mathcal{P}\left(y_{i}^{+}\right)$is increasing for all $y_{i}^{+} \in\left[y_{M_{1}},+\infty\right)$.

For any $y_{i}^{+} \in\left[y_{M_{1}}, y^{*}\right)$, it follows from $y_{i}^{+}<\mathcal{P}\left(y_{i}^{+}\right)<y^{*}$ that $\mathcal{P}^{j}\left(y_{i}^{+}\right)$ is monotonically increasing as $j$ increases, and then $\lim _{j \rightarrow+\infty} \mathcal{P}^{j}\left(y_{i}^{+}\right)=y^{*}$. For any $y_{i}^{+} \in\left(y^{*},+\infty\right]$, we have $y^{*}<\mathcal{P}\left(y_{i}^{+}\right)<y_{i}^{+}$. Thus, based on the monotonicity of $\mathcal{P}\left(y_{i}^{+}\right)$we conclude that $\mathcal{P}^{j}\left(y_{i}^{+}\right)$is monotonically decreasing as $j$ increases, which indicates that $\lim _{j \rightarrow+\infty} \mathcal{P}^{j}\left(y_{i}^{+}\right)=y^{*}$.

For any $y_{i}^{+} \in\left[0, y_{P_{2}}\right] \cup\left[y_{P_{1}}, y_{M_{1}}\right)$, we see that $\mathcal{P}^{l}\left(y_{i}^{+}\right) \in\left[y_{M_{1}},+\infty\right)$ for a given positive integer $l$. Therefore, by employing the same method as used for Theorem 14 we have $\lim _{j \rightarrow+\infty} \mathcal{P}^{l+j}\left(y_{i}^{+}\right)=y^{*}, y_{i}^{+} \in\left[0, y_{P_{2}}\right] \cup\left[y_{P_{1}}, y_{M_{1}}\right)$.

In summary, if $\mathcal{P}\left(y_{M_{i}}\right)>y_{M_{i}}(i=1,2)$, then the unique fixed point $y^{*}$ of the function $\mathcal{P}\left(y_{i}^{+}\right)$is globally stable.

b) If $y_{P_{1}} \leq \mathcal{P}\left(y_{M_{2}}\right)=\mathcal{P}\left(y_{M_{1}}\right) \leq y_{M_{1}}$, then it follows from the inequality $\mathcal{P}\left(y_{P_{1}}\right)>y_{P_{1}}$ that the unique fixed point $y^{*}$ is located in the interval $\left(y_{P_{1}}, y_{M_{1}}\right]$.

Therefore, for case $\left(A_{11}\right)(i i i)$, on the one hand, it follows from $y_{P_{1}} \leq y_{i}^{+}<$ $y^{*}$ and $\mathcal{P}\left(y_{i}^{+}\right)$is decreasing on $\left[y_{P_{1}}, y^{*}\right)$ that we have $\mathcal{P}\left(y_{P_{1}}\right) \geq \mathcal{P}\left(y_{i}^{+}\right)>y^{*}$, furthermore, from $\mathcal{P}^{2}\left(y_{i}^{+}\right)>y_{i}^{+}$for all $y_{i}^{+} \in\left[y_{P_{1}}, y^{*}\right)$ we conclude that $y_{i}^{+}<\mathcal{P}^{2}\left(y_{i}^{+}\right)<y^{*}$. By induction, $\mathcal{P}^{2(j-1)}\left(y_{i}^{+}\right)<\mathcal{P}^{2 j}\left(y_{i}^{+}\right)<y^{*}$ for al$1 j \geq 1$ is true, which indicates that $\mathcal{P}^{2 j}\left(y_{i}^{+}\right)$is monotonically increasing, and $\lim _{j \rightarrow+\infty} \mathcal{P}^{2 j}\left(y_{i}^{+}\right)=y^{*}, y_{i}^{+} \in\left[y_{P_{1}}, y^{*}\right)$.

On the other hand, if $y_{P_{1}} \leq \mathcal{P}\left(y_{M_{2}}\right)=\mathcal{P}\left(y_{M_{1}}\right) \leq y_{M_{1}}$, for any $y_{i}^{+} \in$ $\left[0, y_{P_{2}}\right] \cup\left(y^{*},+\infty\right)$, there must exist a positive integer $l$ such that $\mathcal{P}^{l}\left(y_{i}^{+}\right) \in$ $\left[y_{P_{1}}, y^{*}\right]$, which indicates that $\lim _{j \rightarrow+\infty} \mathcal{P}^{l+2 j}\left(y_{i}^{+}\right)=y^{*}$ for all $y_{i}^{+} \in\left[0, y_{P_{2}}\right] \cup$ $\left(y^{*},+\infty\right)$ monotonically.

Therefore, if $y_{P_{1}} \leq \mathcal{P}\left(y_{M_{2}}\right)=\mathcal{P}\left(y_{M_{1}}\right) \leq y_{M_{1}}$, then the fixed point $y^{*}$ is globally stable provided that $\mathcal{P}^{2}\left(y_{i}^{+}\right)>y_{i}^{+}$for $y_{i}^{+} \in\left[y_{P_{1}}, y^{*}\right)$ is true. This completes the proof.

Theorem 16 For case $\left(A_{12}\right)(i)$, if the fixed point $y^{*}$ is unique for the Poincaré map $\mathcal{P}\left(y_{i}^{+}\right)$, then we have that

a) if $\mathcal{P}\left(y_{Q_{0}}\right)<y_{Q_{0}}$, the unique fixed point $y^{*}$ is globally stable.

b) if $\mathcal{P}\left(y_{Q_{0}}\right)>y_{Q_{0}}$, the fixed point $y^{*}$ is globally stable provided that $\mathcal{P}^{2}\left(y_{i}^{+}\right)>y_{i}^{+}$for $y_{i}^{+} \in\left[y_{Q_{0}}, y^{*}\right)$. 
Proof. Based on Theorem 10, we know that the fixed point of the Poincaré map $\mathcal{P}\left(y_{i}^{+}\right)$exists for case $\left(A_{12}\right)(i)$. Further, if we assume that $y^{*}$ is unique, then the global stability can be shown as follows:

a) if $\mathcal{P}\left(y_{Q_{0}}\right)<y_{Q_{0}}$, for any $y_{i}^{+} \in\left[0, y^{*}\right)$ we have $y_{i}^{+}<\mathcal{P}\left(y_{i}^{+}\right)<y^{*}$, which indicates that $\mathcal{P}^{j}\left(y_{i}^{+}\right)$is monotonically increasing as $j$ increases and $\lim _{j \rightarrow+\infty} \mathcal{P}^{j}\left(y_{i}^{+}\right)=y^{*}$. For any $y_{i}^{+} \in\left(y^{*},+\infty\right]$, we consider two cases: (1) for any $y_{i}^{+} \in\left(y^{*}, y_{Q_{0}}\right]$, it follows from $y^{*}<\mathcal{P}\left(y_{i}^{+}\right)<y_{i}^{+}$and monotonicity of $\mathcal{P}\left(y_{i}^{+}\right)$that we have $y^{*}<\mathcal{P}^{j}\left(y_{i}^{+}\right)<\mathcal{P}^{j-1}\left(y_{i}^{+}\right)$for all $j \geq 1$, which indicates that $\lim _{j \rightarrow+\infty} \mathcal{P}^{j}\left(y_{i}^{+}\right)=y^{*}$. (2) for any $y_{i}^{+} \in\left(y_{Q_{0}},+\infty\right)$, there must be $\mathcal{P}\left(y_{i}^{+}\right) \in\left(0, y_{Q_{0}}\right)$, which indicates that $\lim _{j \rightarrow+\infty} \mathcal{P}^{1+j}\left(y_{i}^{+}\right)=y^{*}$. Thus, the results shown in case $a)$ are true.

b) if $\mathcal{P}\left(y_{Q_{0}}\right)>y_{Q_{0}}$, we consider three intervals: (1) $y_{i}^{+} \in\left[y_{Q_{0}}, y^{*}\right) ;(2) y_{i}^{+} \in$ $\left[0, y_{Q_{0}}\right) ;(3) y_{i}^{+} \in\left(y^{*},+\infty\right)$. For case $(1)$, it follows from $y_{Q_{0}} \leq y_{i}^{+}<y^{*}$ and the Poincaré map $\mathcal{P}\left(y_{i}^{+}\right)$is monotonicity decreasing that we have $\mathcal{P}\left(y_{Q_{0}}\right) \geq$ $\mathcal{P}\left(y_{i}^{+}\right)>y^{*}$, furthermore, $\mathcal{P}^{2}\left(y_{i}^{+}\right)>y_{i}^{+}$for all $y_{i}^{+} \in\left[y_{Q_{0}}, y^{*}\right)$, then $y_{i}^{+}<$ $\mathcal{P}^{2}\left(y_{i}^{+}\right)<y^{*}$ is true. By induction, we conclude that $\mathcal{P}^{2(j-1)}\left(y_{i}^{+}\right)<\mathcal{P}^{2 j}\left(y_{i}^{+}\right)<$ $y^{*}$ for all $j \geq 1$, which means that $\mathcal{P}^{2 j}\left(y_{i}^{+}\right)$is monotonically increasing, and $\lim _{j \rightarrow+\infty} \mathcal{P}^{2 j}\left(y_{i}^{+}\right)=y^{*}$.

For case (2) and case (3), by using similar methods we can show that there exists a positive integer $n$ such that $\mathcal{P}^{n}\left(y_{i}^{+}\right) \in\left[y_{Q_{0}}, y^{*}\right]$, then according to case (1), we see that the results shown in case $b$ ) are true. The proof is completed.

Theorem 17 For case $\left(A_{12}\right)(i i)$, if the fixed point $y^{*}$ is unique, then we have that

a) $y^{*}$ is globally stable if $\mathcal{P}\left(y_{m_{i}}\right)>y_{m_{i}}(i=1,2)$

b) $y^{*}$ is globally stable if $\mathcal{P}\left(y_{m_{i}}\right)<y_{m_{i}}(i=1,2)$ and $\mathcal{P}^{2}\left(y_{i}^{+}\right)<y_{i}^{+}$for $y_{i}^{+} \in\left(y^{*}, y_{m_{2}}\right]$.

c) if $\mathcal{P}\left(y_{Q_{0}}\right)>y_{Q_{0}}, \mathcal{P}\left(y_{m_{2}}\right)>y_{m_{2}}$ and $\mathcal{P}\left(y_{m_{1}}\right)<y_{m_{1}}$, then $y^{*}$ is globally stable provided that $y^{*}<\mathcal{P}^{2}\left(y_{i}^{+}\right)<y_{i}^{+}$for $y_{i}^{+} \in\left(y^{*}, y_{m_{1}}\right]$.

d) if $\mathcal{P}\left(y_{Q_{0}}\right)<y_{Q_{0}}, \mathcal{P}\left(y_{m_{2}}\right)>y_{m_{2}}$ and $\mathcal{P}\left(y_{m_{1}}\right)<y_{m_{1}}, y^{*}$ is globally stable.

Proof. Based on Theorem 11, we know that the fixed point of the $\mathcal{P}\left(y_{i}^{+}\right)$ exists for case $\left(A_{12}\right)(i i)$. Further, if $y^{*}$ is unique, then the global stability can be shown as follows:

$a)$ if $\mathcal{P}\left(y_{m_{i}}\right)>y_{m_{i}}(i=1,2)$, we consider three intervals: (1) $y_{i}^{+} \in\left[y_{m_{1}}, y^{*}\right)$; (2) $y_{i}^{+} \in\left(y^{*},+\infty\right)$; (3) $y_{i}^{+} \in\left(0, y_{m_{1}}\right)$.

For any $y_{i}^{+} \in\left[y_{m_{1}}, y^{*}\right)$, it follows from $y_{i}^{+}<\mathcal{P}\left(y_{i}^{+}\right)<y^{*}$ and $\mathcal{P}\left(y_{i}^{+}\right)$is increasing on $\left[y_{m_{1}},+\infty\right)$ that we have $\mathcal{P}\left(y_{i}^{+}\right)<\mathcal{P}^{2}\left(y_{i}^{+}\right)<y^{*}$. By induction, we conclude that $\mathcal{P}^{j-1}\left(y_{i}^{+}\right)<\mathcal{P}^{j}\left(y_{i}^{+}\right)<y^{*}$ for all $j \geq 1$, which means that $\mathcal{P}^{j}\left(y_{i}^{+}\right)$ is monotonically increasing, and $\lim _{j \rightarrow+\infty} \mathcal{P}^{j}\left(y_{i}^{+}\right)=y^{*}, y_{i}^{+} \in\left[y_{m_{1}}, y^{*}\right)$.

For any $y_{i}^{+} \in\left(y^{*},+\infty\right)$, according to $y^{*}<\mathcal{P}\left(y_{i}^{+}\right)<y_{i}^{+}$and the monotonicity of $\mathcal{P}\left(y_{i}^{+}\right)$, we have $y^{*}<\mathcal{P}^{2}\left(y_{i}^{+}\right)<\mathcal{P}\left(y_{i}^{+}\right)$, which indicates that $\mathcal{P}^{j}\left(y_{i}^{+}\right)$is monotonically decreasing, and $\lim _{j \rightarrow+\infty} \mathcal{P}^{j}\left(y_{i}^{+}\right)=y^{*}, y_{i}^{+} \in\left(y^{*},+\infty\right)$.

For any $y_{i}^{+} \in\left(0, y_{m_{1}}\right)$, there must be $\mathcal{P}\left(y_{i}^{+}\right) \in\left(y_{m_{1}},+\infty\right)$, we obtain $\lim _{j \rightarrow+\infty} \mathcal{P}^{1+j}\left(y_{i}^{+}\right)=y^{*}, y_{i}^{+} \in\left(0, y_{m_{1}}\right)$ based on the previous results. Therefore, the results shown in case $a$ ) are true. 
b) if $\mathcal{P}\left(y_{m_{i}}\right)<y_{m_{i}}(i=1,2)$, then we consider two cases: (1) $y_{i}^{+} \in\left(y^{*}, y_{m_{2}}\right]$; (2) $y_{i}^{+} \in\left(0, y^{*}\right) \cup\left(y_{m_{2}},+\infty\right)$.

For any $y_{i}^{+} \in\left(y^{*}, y_{m_{2}}\right]$, it follows from the monotonicity of $\mathcal{P}\left(y_{i}^{+}\right)$and $\mathcal{P}^{2}\left(y_{i}^{+}\right)<y_{i}^{+}$for $y_{i}^{+} \in\left(y^{*}, y_{m_{2}}\right]$ that we have $y^{*}<\mathcal{P}^{4}\left(y_{i}^{+}\right)<\mathcal{P}^{2}\left(y_{i}^{+}\right)$. By induction, we have $y^{*}<\mathcal{P}^{2 j}\left(y_{i}^{+}\right)<\mathcal{P}^{2(j-1)}\left(y_{i}^{+}\right)$for all $j \geq 1$, which means that $\mathcal{P}^{2 j}\left(y_{i}^{+}\right)$is monotonically decreasing, and $\lim _{j \rightarrow+\infty} \mathcal{P}^{2 j}\left(y_{i}^{+}\right)=y^{*}, y_{i}^{+} \in\left(y^{*}, y_{m_{2}}\right]$.

For any $y_{i}^{+} \in\left(0, y^{*}\right) \cup\left(y_{m_{2}},+\infty\right)$, there has a positive integer $k$ such that $\mathcal{P}^{k}\left(y_{i}^{+}\right) \in\left[y^{*}, y_{m_{2}}\right)$, then we obtain that $\lim _{j \rightarrow+\infty} \mathcal{P}^{k+2 j}\left(y_{i}^{+}\right)=y^{*}, y_{i}^{+} \in$ $\left(0, y^{*}\right) \cup\left(y_{m_{2}},+\infty\right)$. All these results confirm that those shown in case $\left.b\right)$ are true.

c) Similarly, we still consider two cases: (1) $y_{i}^{+} \in\left(y^{*}, y_{m_{1}}\right] ;(2) y_{i}^{+} \in\left(0, y^{*}\right) \cup$ $\left(y_{m_{1}},+\infty\right)$.

For any $y_{i}^{+} \in\left(y^{*}, y_{m_{1}}\right]$, the monotonicity of $\mathcal{P}\left(y_{i}^{+}\right)$in this interval is decreasing, furthermore, as $y^{*}<\mathcal{P}^{2}\left(y_{i}^{+}\right)<y_{i}^{+}$for $y_{i}^{+} \in\left(y^{*}, y_{m_{1}}\right]$, it is easy to know that $y^{*}<\mathcal{P}^{4}\left(y_{i}^{+}\right)<\mathcal{P}^{2}\left(y_{i}^{+}\right)$. By induction, we have $y^{*}<\mathcal{P}^{2 j}\left(y_{i}^{+}\right)<$ $\mathcal{P}^{2(j-1)}\left(y_{i}^{+}\right)$for all $j \geq 1$, which indicates that $\mathcal{P}^{2 j}\left(y_{i}^{+}\right)$is monotonically decreasing with $\lim _{j \rightarrow+\infty} \mathcal{P}^{2 j}\left(y_{i}^{+}\right)=y^{*}, y_{i}^{+} \in\left(y^{*}, y_{m_{1}}\right]$.

For any $y_{i}^{+} \in\left(0, y^{*}\right) \cup\left(y_{m_{1}},+\infty\right)$, similarly there exists a positive integer $l$ such that $\mathcal{P}^{l}\left(y_{i}^{+}\right) \in\left[y^{*}, y_{m_{1}}\right]$. Thus, we have $\lim _{j \rightarrow+\infty} \mathcal{P}^{l+2 j}\left(y_{i}^{+}\right)=y^{*}, y_{i}^{+} \in$ $\left(0, y^{*}\right) \cup\left(y_{m_{1}},+\infty\right)$, which means that the results shown in case $\left.c\right)$ are true.

d) Again, two cases should be considered: (1) $y_{i}^{+} \in\left[y_{m_{2}}, y_{Q_{0}}\right]$; (2) $y_{i}^{+} \in$ $\left(0, y_{m_{2}}\right) \cup\left(y_{Q_{0}},+\infty\right)$.

For the interval $\left[y_{m_{2}}, y_{Q_{0}}\right]$, according to the monotonicity of $\mathcal{P}\left(y_{i}^{+}\right)$, it is easy to know that $\mathcal{P}^{j_{1}}\left(y_{i}^{+}\right)$is monotonically increasing with $\lim _{j_{1} \rightarrow+\infty} \mathcal{P}^{j_{1}}\left(y_{i}^{+}\right)=y^{*}$, $y_{i}^{+} \in\left[y_{m_{2}}, y^{*}\right), \mathcal{P}^{j_{2}}\left(y_{i}^{+}\right)$is monotonically decreasing with $\lim _{j_{2} \rightarrow+\infty} \mathcal{P}^{j_{2}}\left(y_{i}^{+}\right)=y^{*}$, $y_{i}^{+} \in\left(y^{*}, y_{Q_{0}}\right]$.

For any $y_{i}^{+} \in\left(0, y_{m_{2}}\right) \cup\left(y_{Q_{0}},+\infty\right)$, there also exists a positive integer $k$ such that $\mathcal{P}^{k}\left(y_{i}^{+}\right) \in\left[y_{m_{2}}, y_{Q_{0}}\right]$ and we have $\lim _{j_{1} \rightarrow+\infty} \mathcal{P}^{k+j_{1}}\left(y_{i}^{+}\right)=y^{*}$ or $\lim _{j_{2} \rightarrow+\infty} \mathcal{P}^{k+j_{2}}\left(y_{i}^{+}\right)=y^{*}, y_{i}^{+} \in\left(0, y_{m_{2}}\right) \cup\left(y_{Q_{0}},+\infty\right)$. Therefore, the results shown in case $d$ ) are true. This completes the proof.

Theorem 18 For case $\left(A_{12}\right)($ iii), there exists at least one fixed point. Moreover, if we assume that there is only one fixed point, then for the global stability we have:

a) if $\mathcal{P}\left(y_{Q_{0}}\right)>y_{Q_{0}}$, the unique fixed point $y^{*}$ is globally stable.

b) if $\mathcal{P}\left(y_{Q_{0}}\right)<y_{Q_{0}}$, the unique fixed point $y^{*}$ is globally stable provided that $\mathcal{P}^{2}\left(y_{i}^{+}\right)<y_{i}^{+}$for $y_{i}^{+} \in\left(y^{*}, y_{Q_{0}}\right]$.

Proof. Based on Theorem 10, we know that the fixed point of the $\mathcal{P}\left(y_{i}^{+}\right)$ exists for case $\left(A_{1}\right)\left(A_{12}\right)(i i i)$. If we assume that there is only one fixed point, then we can discuss the global stability as follows:

a) From Theorem 7 we know that the Poincaré map is increasing on $\left[y_{Q_{0}},+\infty\right)$, and decreasing on $\left[0, y_{Q_{0}}\right]$. if $\mathcal{P}\left(y_{Q_{0}}\right)>y_{Q_{0}}$, then the unique fixed point $y^{*}>y_{Q_{0}}$, for any $y_{i}^{+} \in\left[y_{Q_{0}}, y^{*}\right)$, and it is easy to know that $\mathcal{P}^{j_{1}}\left(y_{i}^{+}\right)$is 
monotonically increasing with $\lim _{j_{1} \rightarrow+\infty} \mathcal{P}^{j_{1}}\left(y_{i}^{+}\right)=y^{*}, y_{i}^{+} \in\left[y_{Q_{0}}, y^{*}\right) ; \mathcal{P}^{j_{2}}\left(y_{i}^{+}\right)$ is monotonically decreasing with $\lim _{j_{2} \rightarrow+\infty} \mathcal{P}^{j_{2}}\left(y_{i}^{+}\right)=y^{*}, y_{i}^{+} \in\left(y^{*},+\infty\right)$.

For any $y_{i}^{+} \in\left(0, y_{Q_{0}}\right)$, the Poincaré map $\mathcal{P}\left(y_{i}^{+}\right) \in\left(y_{Q_{0}},+\infty\right)$, so we have $\lim _{j_{1} \rightarrow+\infty} \mathcal{P}^{1+j_{1}}\left(y_{i}^{+}\right)=y^{*}$ or $\lim _{j_{2} \rightarrow+\infty} \mathcal{P}^{1+j_{2}}\left(y_{i}^{+}\right)=y^{*}, y_{i}^{+} \in\left(0, y_{Q_{0}}\right)$. The unique fixed point $y^{*}$ is globally stable.

b) if $\mathcal{P}\left(y_{Q_{0}}\right)<y_{Q_{0}}$, for any $y_{i}^{+} \in\left(y^{*}, y_{Q_{0}}\right]$, it follows from the monotonicity of $\mathcal{P}\left(y_{i}^{+}\right)$decreasing in the interval $\left[0, y_{Q_{0}}\right]$, and $\mathcal{P}^{2}\left(y_{i}^{+}\right)<y_{i}^{+}$for $y_{i}^{+} \in$ $\left(y^{*}, y_{Q_{0}}\right]$, that we have $y^{*}<\mathcal{P}^{4}\left(y_{i}^{+}\right)<\mathcal{P}^{2}\left(y_{i}^{+}\right)$. By induction, we conclude that $y^{*}<\mathcal{P}^{2 j}\left(y_{i}^{+}\right)<\mathcal{P}^{2(j-1)}\left(y_{i}^{+}\right)$for all $j \geq 1$, which means that $\mathcal{P}^{2 j}\left(y_{i}^{+}\right)$is monotonically decreasing, and $\lim _{j \rightarrow+\infty} \mathcal{P}^{2 j}\left(y_{i}^{+}\right)=y^{*}, y_{i}^{+} \in\left(y^{*}, y_{Q_{0}}\right]$.

For any $y_{i}^{+} \in\left(0, y^{*}\right) \cup\left(y_{Q_{0}},+\infty\right)$, we can show that there exists a positive integer $l$ such that $\mathcal{P}^{l}\left(y_{i}^{+}\right) \in\left(y^{*}, y_{Q_{0}}\right]$, and then we have $\lim _{j \rightarrow+\infty} \mathcal{P}^{l+2 j}\left(y_{i}^{+}\right)=y^{*}$ for all $y_{i}^{+} \in\left(0, y^{*}\right) \cup\left(y_{Q_{0}},+\infty\right)$. The proof is completed.

\subsection{Order-k periodic solutions}

In this subsection, whether the order- $k(k \geq 2)$ periodic solution of system (1) exists or not has been investigated in some cases.

Theorem 19 If $y_{T^{+}}>y_{P_{1}}$ for case $\left(A_{11}\right)(i i)$ (or if $y_{Q_{2}^{+}}<y_{Q_{0}}$ for case $\left.\left(A_{12}\right)(i)\right)$, and if $y_{Q_{2}^{+}}>y_{Q_{0}}$ for case $\left.\left(A_{12}\right)(i i i)\right)$, then system (1) does not exist a periodic solution with order larger than 2.

Proof. If $y_{T^{+}}>y_{P_{1}}$ for case $\left(A_{11}\right)(i i)$, from Theorem 8 we know that there exists an order-1 periodic solution for system (1). Without loss of generality, we assume that the order-1 periodic solution passes through the point $P\left(V_{L}, \eta_{0}\right)$ and $P^{+}\left(\left(1-P_{V_{L}}\right), \eta_{0}^{+}\right)$, the trajectory with initial point $T^{+}$will reach the line $x=V_{L}$ at the point $T_{1}\left(V_{L}, y_{T_{1}}\right)$, in view of the uniqueness of any two solutions, we see that the point $T_{1}$ lies below the point $T$, then the point $T_{1}$ maps to the point $T_{1}^{+}$after a single impulsive effect. If $\frac{\sqrt{\tau \theta}-1}{\theta} \geq \frac{a}{b}$, then we know that the function $G\left(y_{i}\right)=y_{i}^{+}=y_{i}+\frac{\tau}{1+\theta y_{i}}$ is decreasing monotonically with $y_{i}$, it is indicated that the $y_{i}^{+}$decreases monotonously with $y_{i}$ increasing, then the point $T_{1}^{+}$lies above the point $T^{+}$. By induction, we can prove that the point $T_{i+1}^{+}$lies above the point $T_{i}^{+}(i=1,2, \cdots, n)$, which indicated that the series $T_{i}^{+}$is monotonically increasing, i.e.,

$$
y_{T^{+}}<y_{T_{1}^{+}}<y_{T_{2}^{+}}<y_{T_{3}^{+}}<\cdots<y_{T_{i}^{+}}<\cdots<y_{T_{n}^{+}}<\cdots<\eta_{0}^{+}<\tau .
$$

As a result, an order- $k(k \geq 2)$ periodic solution for system (1) does not exist. If $y_{Q_{2}^{+}}<y_{Q_{0}}$ for case $\left(A_{12}\right)(i)$ or if $y_{Q_{2}^{+}}>y_{Q_{0}}$ for case $\left(A_{12}\right)(i i i)$, the conclusions can be similarly proved. This completes the proof.

Theorem 20 If $y_{Q_{2}^{+}}>y_{Q_{0}}$ and $y_{Q_{3}^{+}} \geq y_{Q_{0}}$ for case $\left(A_{12}\right)\left(\right.$ i) (or if $y_{Q_{2}^{+}}<y_{Q_{0}}$ and $y_{Q_{3}^{+}} \leq y_{Q_{0}}$ for case $\left.\left(A_{12}\right)(i i i)\right)$, and if $y_{T^{+}}>y_{P_{1}}$ and $y_{T_{1}^{+}} \geq y_{P_{1}}$ for case $\left.\left(A_{11}\right)(i)\right)$, then no periodic solution with an order greater than or equal to 3 exists for system (1). 
Proof. If $y_{Q_{2}^{+}}>y_{Q_{0}}$ and $y_{Q_{3}^{+}}=y_{Q_{0}}$, we can see that two curves $\widehat{Q_{0} Q_{2}}$ and $\widehat{Q_{2}^{+} Q_{3}}$ form an order-2 periodic solution. If $y_{Q_{3}^{+}}>y_{Q_{0}}$, all the impulsive points starting from the interval $\left[y_{Q_{3}^{+}}, y_{Q_{2}^{+}}\right] \subset Y_{5}$ lie above the point $Q_{3}^{+}$(here $Y_{5}$ is: $\left.\left[\tau, Y_{i s}^{h}+\frac{\tau}{1+Y_{i s}^{h}}\right]\right)$, in view of the uniqueness of any two solutions, it is easy to get $y_{Q_{0}}<y_{Q_{3}^{+}}<y_{Q_{5}^{+}}<y_{Q_{4}^{+}}<y_{Q_{2}^{+}}=\mathcal{P}\left(y_{Q_{0}}\right)$ for case $\left(A_{12}\right)(i)$, where $y_{Q_{4}^{+}}$ is the vertical component of the initial point $\left(\left(1-P_{V_{L}}\right) V_{L}, y_{Q_{3}^{+}}\right)$from which the solution experiences a single impulsive perturbation. By induction, $y_{Q_{k+1}^{+}}$ is the vertical component of the initial point $\left(\left(1-P_{V_{L}}\right) V_{L}, y_{Q_{k}^{+}}\right)$, from which the solution can only experience a single impulsive perturbation. Therefore, we have the following relations about the pulse points $y_{Q_{i}^{+}}$:

$$
\begin{aligned}
& y_{Q_{0}}<y_{Q_{3}^{+}}<y_{Q_{5}^{+}}< \cdots<y_{Q_{2 n-1}^{+}}<y_{Q_{2 n+1}^{+}}<\cdots< \\
& y_{Q_{2 n+2}^{+}}<y_{Q_{2 n}^{+}}<\cdots<y_{Q_{4}^{+}}<y_{Q_{2}^{+}}=\mathcal{P}\left(y_{Q_{0}}\right) .
\end{aligned}
$$

It follows from (45) that there either exists a unique $y^{*} \in\left[Q_{3}^{+}, Q_{2}^{+}\right]$such that

$$
\lim _{n \rightarrow \infty} y_{Q_{2 n+1}^{+}}=\lim _{n \rightarrow \infty} y_{Q_{2 n}^{+}}=y^{*} .
$$

Or there exist two different values $y_{1}^{*} \neq y_{2}^{*}$ and $y_{1}^{*}, y_{2}^{*} \in\left[Q_{3}^{+}, Q_{2}^{+}\right]$such that

$$
\lim _{n \rightarrow \infty} y_{Q_{2 n+1}^{+}}=y_{1}^{*} \text {, and } \lim _{n \rightarrow \infty} y_{Q_{2 n}^{+}}=y_{2}^{*} .
$$

Therefore, the difference equation shown in Theorem 3 either exists a fixed point or a period two-point cycle, i.e. for system (1) an order- $k(k \geq 3)$ periodic solution does not exist if $y_{Q_{2}^{+}}>y_{Q_{0}}$ and $y_{Q_{3}^{+}} \geq y_{Q_{0}}$ for case $\left(A_{12}\right)(i)$.

If $y_{Q_{2}^{+}}<y_{Q_{0}}$ and $y_{Q_{3}^{+}} \leq y_{Q_{0}}$ for case $\left(A_{12}\right)(i i i)$ or if $y_{T^{+}}>y_{P_{1}}$ and $y_{T_{1}^{+}} \geq y_{P_{1}}$ for case $\left(A_{11}\right)(i)$, the conclusions can also be proved by using the same methods. This completes the proof.

\section{Conclusions}

Lotka-Volterra predator-prey systems with impulsive effects can provide theories and suggest strategies for deploying a variety of control techniques as part of integrated pest management. The two most common manipulations considered in these systems are the spraying of pesticides and releases of natural enemies of the pest. Previously studied impulsive models have assumed that the interventions (impulsive effects) occurred at either fixed times or at unfixed times. In these models, the quantity of natural enemies released was usually taken to be a constant number[21-27]. However, factors such as resource limitation, feedback from monitoring data, and management goals were ignored. Consequently, assumptions about the methods used in interventions should be more realistic for real world applications. Therefore, we take the economic threshold level for guiding the implementation of the integrated control tactics, 
i.e. once the density of the pests arrives the critical size $V_{L}$, density dependent control interventions including spraying pesticides and releasing natural enemies could be applied immediately. Based on these facts, we proposed a novel Lotka-Volterra predator-prey model here concerning density guided quantities of natural enemies released with a state-dependent feedback control strategy. One of the main purposes was to provide a complete qualitative analysis for system (1), and show how nonlinear pulse functions affect the dynamics of the system.

Firstly, we reviewed the properties of the ODE model (2) with its first integral by using the Lambert W function. Subsequently, in order to define the impulsive sets and phase sets, we analyzed the effects of the key parameters: $\delta, V_{L}$ and $h$ on the signs of $A_{h} ; \tau$ and $\theta$ for the key value $\frac{\sqrt{\tau \theta}-1}{\theta}$. If $A_{h} \geq 0$, the impulsive set is defined by $M$, and any solution with initial value $\left(x_{0}^{+}, y_{0}^{+}\right) \in$ $\mathcal{N}$ with $y_{0}^{+} \in\left(Y_{\min }^{h}, Y_{\max }^{h}\right)$ does not experience impulsive perturbations. In contrast, if $A_{h}<0$, the impulsive set is defined by $M_{0}$, as shown in Lemma 1 and Lemma 2. The detailed domains of the impulsive and phase sets of system (1) are described in Table 1, from which the analytical formula for Poincaré map has been provided, as shown in Theorem 3 and Table 2. Note that as the quantity of natural enemies released is not a fixed number, the nonlinear term $\frac{\tau}{1+\theta y(t)}$ and the value of $\frac{\sqrt{\tau \theta}-1}{\theta}$ not only result in difficulties for the construction of the Poincaré map, but also add complexity to the analysis of the periodic solution for the system.

In particular, it can be seen from Theorem 4 that if the quantity of natural enemies released is zero $(\tau=0$ here), then the dynamics of system (1) strictly depend on the sign of $A_{h}$. If the quantity $A_{h}$ related to threshold $V_{L}$ is equal to zero, then both the pest and natural enemy populations could coexist, i.e. any solution could be an order- 1 periodic solution once the initial point lies in the phase set; If $A_{h}<0$ then the boundary order-1 periodic solution is globally stable and the natural enemies will die out eventually; If $A_{h}>0$ then the boundary order- 1 periodic solution becomes unstable, and system (1) could present a positive order-1 periodic solution, as shown in Fig. 3. Furthermore, the dynamics of system (1) for $\tau>0$ have been investigated in more detail in the present paper. The main results reveal that the nonlinear control strategies which depend on the densities of both populations will cause different complex dynamics. That is, variations in the densities of both populations will result in changes to the impulsive functions, thereby generating difficulties and complexities in analyzing the dynamics of system (1).

All the theoretical results shown in the present paper reveal that the nonlinear impulsive control actions are not only more realistic, but also can generate new dynamic behaviour in comparison with those described in previous studies[21, 22, 37]. We can successfully control the pest population such that its density is less than the threshold $V_{L}$ by a finite number of control actions, i.e. the solution of system (1) eventually stabilizes to a periodic solution of the corresponding ODE model. The establishment of impulsive and phase sets and 
the Poincaré map make it easier to have a clear realization about the dynamic behaviour of the system (1).

There are different kinds of functional response functions which have been introduced into the Lotka-Volterra prey-predator system in many previous papers [26, 27, 38, 39], which result in the more realistic and complex LotkaVolterra systems. Therefore, a future research direction will not only be to assess how nonlinear impulsive control actions affect the dynamics of models that include such functional responses, but also it could apply the main methods and techniques developed in the present paper to study other analogous biological systems such as the glucose insulin regulatory system with state dependent feedback control [40].

\section{Acknowledgements}

This work was supported by the National Natural Science Foundation of China (NSFCs, 11471201, 11631012, 61772017), and by the Fundamental Research Funds for the Central Universities GK201701001, and by the Youth Foundation of Hubei University For Nationalities MY2017Q007.

The authors declare no conflict of interest.

\section{References}

1. V. Volterra: Variations and fluctuations of the number of individuals in animal species living together. ICES J. Mar. Sci. 3(1), 3-51 (1928)

2. M.W. Sabelis, O. Diekmann, V.A.A. Jansen: Metapopulation persistence despite local extinction: predator-prey patch models of the Lotka-Volterra type. Biol. J. Linn. Soc. 42, 267-283 (1991)

3. D. S. Boukal, V. Křivan: Lyapunov functions for Lotka-Volterra predatorprey models with optimal foraging behavior. J. Math. Biol. 39, 493-517 (1999)

4. G. Seo., D. L. DeAngelis: A predator-prey model with a Holling type I functional response including a predator mutual interference. J. Nonlinear. Sci. 21, 811-833 (2011)

5. P. Y.H. Pang, M. Wang: Strategy and stationary pattern in a three-species predator-prey model. J. Differ. Equations. 200, 245-273 (2004)

6. M.A. Aziz-Alaoui, M. Daher Okiye: Boundedness and global stability for a predator-prey model with modified Leslie-Gower and Holling-type II schemes. Appl. Math. Lett. 16, 1069-1075 (2003)

7. S. Zhang, X. Meng, T. Zhang: Dynamics analysis and numerical simulations of a stochastic non-autonomous predator-prey system with impulsive effects. Nonlinear Analysis: Hybrid Systems. 26, 19-37 (2017)

8. A.B. Roy, F. Solimano: Global stability and oscillations in classical LotkaVolterra loops. J. Math. Biol. 24, 603-617 (1987). 
9. S. Choo: Global stability in n-dimensional discrete Lotka-Volterra predator-prey models. Adv. Differ. Equ.-NY. 11, 1-17 (2014)

10. E. Beretta, V. Capasso, F. Rinaldi: Global stability results for a generalized Lotka-Volterra system with distributed delays: applications to predator-prey and to epidemic systems. J. Math. Biol. 26, 661-688 (1988)

11. Y. Kuang, H.L. Smith: Global stability for infinite delay Lotka-Volterra type systems. J. Differ. Equations. 103, 221-246 (1993)

12. Y. Li, Y. Kuang: Periodic solutions of periodic delay Lotka-Volterra equations and systems. J. Math. Anal. Appl. 255, 260-280 (2001)

13. G. Zhu, X. Meng, L. Chen: The dynamics of a mutual interference age structured predator-prey model with time delay and impulsive perturbations on predators. Applied Mathematics and Computation. 216(1), 308-316 (2010)

14. B. Wang, J. Yan, J. Cheng, S. Zhong: New criteria of stability analysis for generalized neural networks subject to time-varying delayed signals. Applied Mathematics and Computation. 314, 322-333 (2017)

15. G. Zeng, L. Chen, J. Chen: Persistence and periodic orbits for two-species nonautonomous diffusion Lotka-Volterra models. Math. Comput. Model. 20, 69-80 (1994)

16. F. Cao, L. Chen: Asymptotic behavior of nonautonomous diffusive LotkaVolterra model. System Sci. \& Math. Sci. 11, 107-111 (1998)

17. J. Cui, L. Chen: Permanence and extinction in logistic and Lotka-Volterra systems with diffusion. J. Math. Anal. Appl. 258, 512-535 (2001)

18. A. Hastings: Global Stability in Lotka-Volterra Systems with Diffusion. J. Math. Biol. 6, 163-168 (1978)

19. M. L. Flint, R. van den Bosch: Introduction to Integrated Pest Management. Plenum press, New York (1981)

20. J.C. Van Lenteren: Integrated pest management in protected crops. In: Integrated pest management, pp. 311-320. Chapman Hall, London (1995)

21. S.Y. Tang, L.S. Chen: Modelling and analysis of integrated pest management strategy. Discrete Cont. Dyn.-B. 4, 759-768 (2004)

22. S.Y. Tang, R.A. Cheke: State-dependent impulsive models of integrated pest management (IPM) strategies and their dynamic consequences. J. Math. Biol. 50, 257-292 (2005)

23. S.Y. Tang, Y.N. Xiao, L.S. Chen, R.A. Cheke: Integrated pest management models and their dynamical behaviour. B. Math. Biol. 67, 115-135 (2005)

24. B. Liu, Y.J. Zhang, L.S. Chen, L.H. Sun: The dynamics of a preydependent consumption model concerning integrated pest management. Acta Math. Sin. 21(3), 541-554 (2005)

25. X.N. Liu, L.S. Chen: Complex dynamics of Holling type II LotkaVolterra predator-prey system with impulsive perturbations on the predator. Chaos, Solitons Fract. 16, 311-320 (2003)

26. S.Y. Tang, B. Tang, A.L. Wang, Y.N. Xiao: Holling II predator-prey impulsive semi-dynamic model with complex Poincare map. Nonlinear Dynam. 81, 1579-1596 (2015) 
27. J. Yang, S. Tang: Holling type II predator-prey model with nonlinear pulse as state-dependent feedback control. J. Comput. Appl. Math. 291, 225-241 (2016)

28. L. Feng, Z. Liu: An impulsive periodic predator-prey Lotka-Volterra type dispersal system with mixed functional responses. J. Appl. Math. Comput. 45, 235-257 (2014)

29. S.Y. Tang, W.H. Pang, R.A. Cheke, J.H. Wu: Global dynamics of a state-dependent feedback control system. Adv. Differ. Equ. 2015(1), 322 (2015)

30. X. Wang, Y. Tian, S. Tang: A Holling Type II Pest and Natural Enemy Model with Density Dependent IPM Strategy. Math. Probl. Eng. 2017, 1-12 (2017)

31. R.M. Corless, G.H. Gonnet, D.E.G. Hare, D.J. Jeffrey, D.E. Knuth: On The Lambert W Function. Adv. Comput. Math. 5, 329-359 (1996)

32. K. Ciesielski: On stability in impulsive dynamical systems. Bull. Pol. Acad. Sci. Math. 52, 81-91 (2004)

33. S. Kaul: On impulsive semidynamical systems. J. Math. Anal. Appl. 150, 120-128 (1990)

34. S. Kaul: On impulsive semidynamical systems III: Lyapunov stability. Recent Trends Differ. Equ. Ser. Appl. Anal. 1, 335-345 (1992)

35. D.D. Bainov, P.S. Simeonov: Systems with impulse effect: Stability, Theory and Applications. Ellis Hordwood limited, Chichester (1989)

36. K. Ciesielski: On semicontinuity in impulsive dynamical systems. Bull. Pol. Acad. Sci. Math. 52, 71-80 (2004)

37. W. Gao, S.Y. Tang: The effects of impulsive releasing methods of natural enemies on pest control and dynamical complexity. Nonlinear Anal.Hybri. 5, 540-553 (2011)

38. G. Jiang, Q. Lu, L. Qian: Complex dynamics of a Holling type II preypredator system with state feedback control. Chaos, Solitons Fract. 31, 448-461 (2007)

39. S. Li, W. Liu: A delayed Holling type III functional response predatorprey system with impulsive perturbation on the prey. Adv. Differ. Equ. 2016, 42 (2016)

40. M.Z. Huang, J.X. Li, X.Y. Song, H.J. Guo: Modeling impulsive injections of insulin: Towards aritificial pancreas. SIAM J. Appl. Math. 72, 15241548(2012). 